\title{
Variability of Ventricular Repolarization Dispersion Quantified by Time-Warping the Morphology of the T-waves
}

\author{
Julia Ramírez, Michele Orini, J. Derek Tucker, Esther Pueyo and Pablo Laguna
}

\begin{abstract}
Objective: We propose two electrocardiogram (ECG)-derived markers of T-wave morphological variability in the temporal, $d_{w}$, and amplitude, $d_{a}$, domains. Two additional markers, $d_{w}^{\mathrm{NL}}$ and $d_{a}^{\mathrm{NL}}$, restricted to measure the non-linear information present within $d_{w}$ and $d_{a}$ are also proposed. Methods: We evaluated the accuracy of the proposed markers in capturing T-wave time and amplitude variations in 3 situations: (1) In a simulated set up with presence of additive Laplacian noise, (2) when modifying the spatio-temporal distribution of electrical repolarization with an electro-physiological cardiac model and (3) in ECG records from healthy subjects undergoing a tilt table test. Results: The metrics $d_{w}, d_{a}, d_{w}^{\mathrm{NL}}$ and $d_{a}^{\mathrm{NL}}$ followed T-wave time and amplitude induced variations under different levels of noise, were strongly associated with changes in the spatiotemporal dispersion of repolarization, and showed to provide additional information to differences in the heart rate, $Q T$ and $T_{\mathrm{pe}}$ intervals, and in the T-wave width and amplitude. Conclusion: The proposed ECG-derived markers robustly quantify T-wave morphological variability, being strongly associated with changes in the dispersion of repolarization. Significance: The proposed ECG-derived markers can help to quantify the variability in the dispersion of ventricular repolarization, showing a great potential to be used as arrhythmic risk predictors in clinical situations.
\end{abstract}

Index Terms-Electrocardiogram, morphological variability, repolarization, T-wave, time-warping.

\section{INTRODUCTION}

$\mathbf{V}$ ENTRICULAR arrhythmias are an important cause of morbidity and sudden cardiac death (SCD) in almost all forms of heart disease. Detection and prevention of arrhythmic events are the main strategies to decrease SCD outcomes [1]. Enhanced spatio-temporal repolarization heterogeneity is related to the generation of ventricular arrhythmias that could

This paper was submitted for review on $\mathrm{x}, \mathrm{x}$. This work was supported in part by project TIN2013-41998-R from Spanish Ministry of Economy and Competitiveness (MINECO), Spain, and by Aragón Government, Spain and from European Social Fund (EU) through Biomedical Signal Interpretation and Computational Simulation (BSICoS) group. The computation was performed at the High Performance computing platform of the NANBIOSIS ICTS, Biomedical Research Networking Center in Bioengineering, Biomaterials and Nanomedicine (CIBER-BBN) and Aragón Institute of Engineering Research (I3A), Zaragoza, Spain.

*J. Ramírez is with CIBER-BBN, Zaragoza, Spain, and with BSICoS group, I3A, IIS Aragón, University of Zaragoza, Zaragoza, Spain (correspondence e-mail: Julia.Ramirez@unizar.es). M. Orini is with the Institute of Cardiovascular Science, University College London, London, UK, and with the Barts Heart Centre, London, UK. J. D. Tucker is with Sandia National Laboratories, Albuquerque, NM 87015 USA. E. Pueyo and P. Laguna are with BSICoS group, I3A, IIS Aragón, University of Zaragoza, Zaragoza, Spain, and with CIBER-BBN, Zaragoza, Spain.

Copyright (c) 2016 IEEE. Personal use of this material is permitted. However, permission to use this material for any other purposes must be obtained from the IEEE by sending an email to pubs-permissions@ieee.org. lead to SCD [2], and increased spatio-temporal repolarization variability has been linked to increased arrhythmic risk [3] in both beat-to-beat (short-term) scenarios [4, 5], and when measuring at different stable heart rates $[6,7]$. The T-wave on the electrocardiogram (ECG) reflects the spatiotemporal dispersion of repolarization times of the ventricular myocites, with homogeneous increments of this dispersion being reflected as both linear and non-linear variations of the T-wave width and amplitude, and enhanced heterogeneities being manifested as additional non-linear deformations of the T-wave, such as notches or asymmetries [8-10].

Previous techniques developed to investigate cardiac repolarization from the surface ECG were based on interval repolarization durations and have been shown to reflect spatiotemporal repolarization heterogeneity [11, 12], such as the QT interval, its corrected version [13], T-wave width [14], the distance from the peak to the end of the $\mathrm{T}$ wave $\left(T_{\mathrm{pe}}\right.$ interval) [15], the dynamic changes in the $T_{\mathrm{pe}}$ interval with respect to the heart rhythm [16], the variability of the QT interval [3], the T-wave alternans [17], or the ST elevation [18]. However, these markers might not be able to capture both linear and non-linear T-wave morphological variations, and extracting such information may provide improved ventricular arrhythmic risk. Although this limitation has been faced by recent indices, including the total cosine R-to-T descriptor, the morphology dispersion index or the T-wave residuum [19], they still lack sufficient superiority and they require the information provided by the vectorcardiogram, which could also become a restriction in the clinical practice.

In addition, variations in the temporal domain or misalignments between T-waves might complicate the comparison and corrupt the measurement of amplitude variability. Linear and non-linear temporal re-parameterization (warping) have been used in the literature to overcome this limitation, align ECG waves [20-22] and measure amplitude differences with improved accuracy. However, the warping information has never been used as a marker to assess the variability in the time domain.

The objective of this study is to propose and assess the ability of two ECG-derived markers, $d_{w}$, and $d_{a}$, and their non-linearly restricted versions, $d_{w}^{\mathrm{NL}}$ and $d_{a}^{\mathrm{NL}}$, to quantify singlelead T-wave morphological variability. The point-wise variability between the temporal domains of different T-waves, or the amount of warping needed to remove the time domain variability, is measured by $d_{w}$, while $d_{a}$ quantifies the pointwise amplitude variability after time warping, or the amplitude 


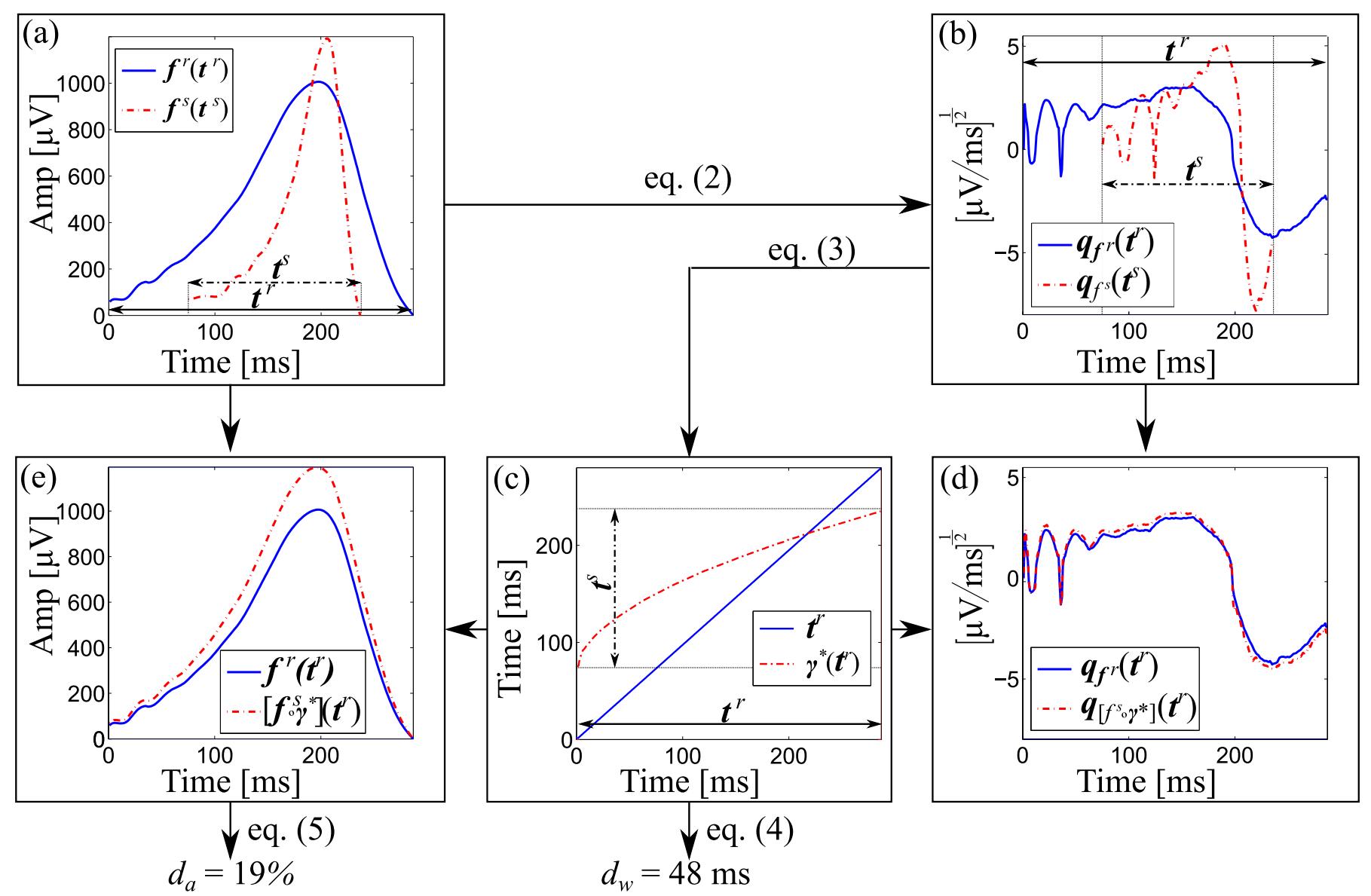

Fig. 1. Diagram flow illustrating the computation of $d_{w}$ and $d_{a}$. (a) Reference T-wave (solid blue) and a T-wave presenting both time and amplitude variability (shorter duration and larger amplitude) (dashed red). (b) Applying eq. (2) we obtain their respective square-root slope functions. (c) Optimizing eq. (3) with the "Dynamic Programming" algorithm (Appendix A), we get $\boldsymbol{\gamma}^{*}\left(\boldsymbol{t}^{r}\right)$, the warping function that optimally relates $\boldsymbol{t}^{r}$ and $\boldsymbol{t}^{s}$. (e) The re-parameterization of $\boldsymbol{f}^{s}\left(\boldsymbol{t}^{s}\right)$ using $\boldsymbol{\gamma}^{*}\left(\boldsymbol{t}^{r}\right)$ leads to $\left[\boldsymbol{f}^{s} \circ \boldsymbol{\gamma}^{*}\right]\left(\boldsymbol{t}^{r}\right)$, the warped T-wave with no remaining time domain variability, and only presenting amplitude variability. (d) Square-root slope functions of the reference (solid blue) and warped (dashed red) T-waves.

variability after removing the temporal domain variability. The markers $d_{w}^{\mathrm{NL}}$ and $d_{a}^{\mathrm{NL}}$ quantify the strictly non-linear warping and amplitude levels within $d_{w}$ and $d_{a}$, respectively. First, the robustness of $d_{w}, d_{a}, d_{w}^{\mathrm{NL}}$ and $d_{a}^{\mathrm{NL}}$ against noise in a simulated set up is evaluated. Next, an electro-physiological cardiac model is used to investigate the relation between $d_{w}$, $d_{a}, d_{w}^{\mathrm{NL}}$ and $d_{a}^{\mathrm{NL}}$ and the morphological changes of the action potential at cellular level. Finally, the T-wave morphological variations produced by a tilt test in real ECG are quantified using $d_{w}, d_{a}, d_{w}^{\mathrm{NL}}$ and $d_{a}^{\mathrm{NL}}$, and their correlation with the heart rate, the QT and $T_{\mathrm{pe}}$ intervals, and the T-wave width (TW) and amplitude (TA) is also studied.

\section{Methods}

\section{A. Mathematical Framework}

The methodology used in this work has been adapted from the mathematical framework presented by Srivastava et al [23]. To exemplify the process of T-wave morphology comparison, consider two T-waves, $\boldsymbol{f}^{r}\left(\boldsymbol{t}^{r}\right)=\left[f^{r}\left(t^{r}(1)\right), \ldots, f^{r}\left(t^{r}\left(N_{r}\right)\right)\right]^{\top}$ and $\boldsymbol{f}^{s}\left(\boldsymbol{t}^{s}\right)=\left[f^{s}\left(t^{s}(1)\right), \ldots, f^{s}\left(t^{s}\left(N_{s}\right)\right)\right]^{\top}$, where $\boldsymbol{t}^{r}=$ $\left[t^{r}(1), \ldots, t^{r}\left(N_{r}\right)\right]^{\top}$ and $\boldsymbol{t}^{s}=\left[t^{s}(1), \ldots, t^{s}\left(N_{s}\right)\right]^{\top}$ and $N_{r}$ and $N_{s}$ being the total duration of $\boldsymbol{t}^{r}$ and $\boldsymbol{t}^{s}$, respectively, as illustrated in Figure 1(a). We take $\boldsymbol{f}^{r}\left(\boldsymbol{t}^{r}\right)$ as the reference Twave and $f^{s}\left(\boldsymbol{t}^{s}\right)$ as the T-wave to be compared with respect to $\boldsymbol{f}^{r}\left(\boldsymbol{t}^{r}\right)$.

Let $\gamma\left(\boldsymbol{t}^{r}\right)$ be the warping function that relates $\boldsymbol{t}^{r}$ and $\boldsymbol{t}^{s}$, such that the composition $\left[\boldsymbol{f}^{s} \circ \boldsymbol{\gamma}\right]\left(\boldsymbol{t}^{r}\right)=\boldsymbol{f}^{s}\left(\boldsymbol{\gamma}\left(\boldsymbol{t}^{r}\right)\right)$ denotes the re-parameterization or time domain warping of $\boldsymbol{f}^{s}\left(\boldsymbol{t}^{s}\right)$ using $\gamma\left(\boldsymbol{t}^{r}\right)$, i.e. $\boldsymbol{f}^{s}\left(\gamma\left(\boldsymbol{t}^{r}\right)\right)$ represents the amplitude values of $\boldsymbol{f}^{s}\left(\boldsymbol{t}^{s}\right)$ if its temporal vector was $\boldsymbol{t}^{r}$. A traditional way to find the optimal warping function is:

$$
\boldsymbol{\gamma}^{*}\left(\boldsymbol{t}^{r}\right)=\underset{\gamma\left(\boldsymbol{t}^{r}\right)}{\arg \min }\left\{\left\|\boldsymbol{f}^{r}\left(\boldsymbol{t}^{r}\right)-\boldsymbol{f}^{s}\left(\boldsymbol{\gamma}\left(\boldsymbol{t}^{r}\right)\right)\right\|\right\} .
$$

However, this metric has some limitations. Since it is not symmetric $\left(\left\|\boldsymbol{f}^{r}\left(\boldsymbol{t}^{r}\right)-\boldsymbol{f}^{s}\left(\boldsymbol{\gamma}\left(\boldsymbol{t}^{r}\right)\right)\right\| \neq\left\|\boldsymbol{f}^{s}\left(\boldsymbol{t}^{s}\right)-\boldsymbol{f}^{r}\left(\boldsymbol{\gamma}^{-1}\left(\boldsymbol{t}^{s}\right)\right)\right\|\right)$, it is not a proper distance and can lead to degenerate results if $\boldsymbol{f}^{r}\left(\boldsymbol{t}^{r}\right)$ and $\boldsymbol{f}^{s}\left(\boldsymbol{t}^{s}\right)$ present variations in the amplitude domain ("pinching effect") [24]. To address this, the square-root slope function (SRSF) was proposed [23, 25] to solve the warping in a well-defined geometrical space by warping, instead, the SRSFs, defined as the square-root of the derivative of $\boldsymbol{f}(\boldsymbol{t})$, considering the sign:

$$
\boldsymbol{q}_{f}(\boldsymbol{t})=\operatorname{sign}(\dot{\boldsymbol{f}}(\boldsymbol{t})) \sqrt{|\dot{\boldsymbol{f}}(\boldsymbol{t})|}
$$


The SRSF of $\boldsymbol{f}^{r}\left(\boldsymbol{t}^{r}\right)$ and $\boldsymbol{f}^{s}\left(\boldsymbol{t}^{s}\right), \boldsymbol{q}_{f^{r}}\left(\boldsymbol{t}^{r}\right)$ and $\boldsymbol{q}_{f^{s}}\left(\boldsymbol{t}^{s}\right)$, respectively, are shown in Figure 1 (b). Now, the optimal warping function is the one that minimizes the amplitude difference between the SRSF of $\boldsymbol{f}^{r}\left(\boldsymbol{t}^{r}\right)$ and $\boldsymbol{f}^{s}\left(\boldsymbol{\gamma}\left(\boldsymbol{t}^{r}\right)\right), \boldsymbol{q}_{f^{r}}\left(\boldsymbol{t}^{r}\right)$ and $\boldsymbol{q}_{\left[f^{s} \circ \gamma\right]}\left(\boldsymbol{t}^{r}\right)=\boldsymbol{q}_{f^{s}}\left(\gamma\left(\boldsymbol{t}^{r}\right)\right) \sqrt{\dot{\gamma}\left(\boldsymbol{t}^{r}\right)}$, respectively [23, 25]:

$$
\begin{aligned}
\boldsymbol{\gamma}^{*}\left(\boldsymbol{t}^{r}\right) & =\underset{\gamma\left(\boldsymbol{t}^{r}\right)}{\arg \min }\left(\left\|\boldsymbol{q}_{f^{r}}\left(\boldsymbol{t}^{r}\right)-\boldsymbol{q}_{\left[f^{s} \circ \gamma\right]}\left(\boldsymbol{t}^{r}\right)\right\|\right) \\
& =\underset{\boldsymbol{\gamma}\left(\boldsymbol{t}^{r}\right)}{\arg \min }\left(\left\|\boldsymbol{q}_{f^{r}}\left(\boldsymbol{t}^{r}\right)-\boldsymbol{q}_{f^{s}}\left(\boldsymbol{\gamma}\left(\boldsymbol{t}^{r}\right)\right) \sqrt{\dot{\gamma}\left(\boldsymbol{t}^{r}\right)}\right\|\right) .
\end{aligned}
$$

The dynamic programming algorithm, explained in Appendix A, was used to obtain the solution of this optimization [26]. The metric minimized in (3) is a proper distance, which overcomes the limitation of the metric minimized in (1) $[23,25,27]$. The optimal warping function, $\gamma^{*}\left(\boldsymbol{t}^{r}\right)$, that optimally warps $\boldsymbol{f}^{r}\left(\boldsymbol{t}^{r}\right)$ and $\boldsymbol{f}^{s}\left(\boldsymbol{t}^{s}\right)$ is shown in Figure 1 (c). The warped T-wave, $\boldsymbol{f}^{s}\left(\boldsymbol{\gamma}^{*}\left(\boldsymbol{t}^{r}\right)\right)$ is shown in Figure 1 (e), together with the reference T-wave, $f^{r}\left(t^{r}\right)$, while their corresponding SRSFs are shown in Figure 1 (d).

The level of warping may be different under various situations and it reflects important information regarding time domain variability. We define a metric, $d_{w}$, that quantifies the level of warping needed to optimally align any two T-waves as the average of the absolute difference value between $\gamma^{*}\left(\boldsymbol{t}^{r}\right)$ and $\boldsymbol{t}^{r}$ :

$$
d_{w}=\frac{1}{N_{r}} \sum_{n=1}^{N_{r}}\left|\gamma^{*}\left(t^{r}(n)\right)-t^{r}(n)\right|,
$$

If $\gamma^{*}\left(\boldsymbol{t}^{r}\right)=\boldsymbol{t}^{r}$, then the functions are perfectly aligned, so it makes sense to use the difference between $\gamma^{*}\left(\boldsymbol{t}^{r}\right)$ and $\boldsymbol{t}^{r}$ to quantify the variability between each T-wave time domain. Figure 1 shows the value of $d_{w}$ quantifying the time variability between $\boldsymbol{f}^{r}\left(\boldsymbol{t}^{r}\right)$ and $\boldsymbol{f}^{s}\left(\boldsymbol{t}^{s}\right)$. Once the time domain variability has been compensated for by optimally warping $\boldsymbol{f}^{s}\left(\boldsymbol{t}^{s}\right)$, the remaining variability is merely amplitude variability, as shown in Figure 1 (e). Therefore, the amplitude difference between $\boldsymbol{f}^{r}\left(\boldsymbol{t}^{r}\right)$ and $\boldsymbol{f}^{s}\left(\boldsymbol{\gamma}^{*}\left(\boldsymbol{t}^{r}\right)\right)$, i.e. the variability between $\boldsymbol{f}^{r}\left(\boldsymbol{t}^{r}\right)$ and $\boldsymbol{f}^{s}\left(\boldsymbol{t}^{s}\right)$ not due to deviations in the time domain, is quantified as the area contained between $\boldsymbol{f}^{r}\left(\boldsymbol{t}^{r}\right)$ and $\boldsymbol{f}^{s}\left(\boldsymbol{\gamma}^{*}\left(\boldsymbol{t}^{r}\right)\right)$, normalized by the L2-norm of $\boldsymbol{f}^{r}\left(\boldsymbol{t}^{r}\right)$ :

$$
d_{a}=\frac{e_{a}}{\left\|e_{a}\right\|} \cdot \frac{\left\|\boldsymbol{f}^{s}\left(\boldsymbol{\gamma}^{*}\left(\boldsymbol{t}^{r}\right)\right)-\boldsymbol{f}^{r}\left(\boldsymbol{t}^{r}\right)\right\|}{\left\|\boldsymbol{f}^{r}\left(\boldsymbol{t}^{r}\right)\right\|} \times 100,
$$

where $\frac{e_{a}}{\left\|e_{a}\right\|}, e_{a}=\sum_{n=1}^{N_{r}}\left(f^{s}\left(\gamma^{*}\left(t^{r}\right)\right)-f^{r}\left(t^{r}\right)\right)$ accounts for the sign. Figure 1 shows the value of $d_{a}$, measuring the amplitude variability between $\boldsymbol{f}^{r}\left(\boldsymbol{t}^{r}\right)$ and $\boldsymbol{f}^{s}\left(\boldsymbol{t}^{s}\right)$.

\section{B. Extracting Non-linear T-wave Morphological Variability}

The proposed markers, $d_{w}$ and $d_{a}$, contain information about linear and non-linear differences in both time and amplitude, respectively. Isolating the strictly non-linear variability information might provide additional understanding on the different sources generating the morphology of the T-wave.

Figure 2, panel (a), shows the optimal warping function from Figure 1, panel (c). As explained in equation (4), the marker $d_{w}$ is calculated as the mean deviation of $\boldsymbol{\gamma}^{*}\left(\boldsymbol{t}^{r}\right)$ from $\boldsymbol{t}^{r}$, marked in yellow in Figure 2, panel (a). By fitting $\boldsymbol{\gamma}^{*}\left(\boldsymbol{t}^{r}\right)$ with a linear regression, $\gamma_{l}^{*}\left(\boldsymbol{t}^{r}\right)$, (black dashed line), and measuring the mean deviation of $\gamma^{*}\left(\boldsymbol{t}^{r}\right)$ with respect to this regression, we can have a quantification of the level of nonlinear warping (dashed cyan region). This can be expressed as:

$$
d_{w}^{\mathrm{NL}}=\frac{1}{N_{r}} \sum_{n=1}^{N_{r}}\left|\gamma^{*}\left(t^{r}(n)\right)-\gamma_{l}^{*}\left(t^{r}(n)\right)\right|,
$$

where $\gamma_{l}^{*}\left(\boldsymbol{t}^{r}\right)$ is the linear fitting of $\gamma^{*}\left(\boldsymbol{t}^{r}\right)$ using the least absolute residual method [28] (Figure 2 (a)). The linear warping can be quantified by measuring the mean deviation of $\gamma_{l}^{*}\left(\boldsymbol{t}^{r}\right)$ from $\boldsymbol{t}^{r}$ (non-dashed region in Figure 2, panel a).

Regarding $d_{a}$, normalizing the warped T-waves, we can quantify non-linear amplitude differences not due to homogeneous scaling, and possibly caused by heterogeneous dispersion of repolarization times:

$$
d_{a}^{\mathrm{NL}}=\left\|\frac{\boldsymbol{f}^{r}\left(\boldsymbol{t}^{r}\right)}{\left\|\boldsymbol{f}^{r}\left(\boldsymbol{t}^{r}\right)\right\|}-\frac{\boldsymbol{f}^{s}\left(\boldsymbol{\gamma}^{*}\left(\boldsymbol{t}^{r}\right)\right)}{\left\|\boldsymbol{f}^{s}\left(\boldsymbol{\gamma}^{*}\left(\boldsymbol{t}^{r}\right)\right)\right\|}\right\| \times 100 .
$$

This is depicted in Figure 2 (b). Panel (b.1) shows the reference, $\boldsymbol{f}^{r}\left(\boldsymbol{t}^{r}\right)$ (solid blue), and studied, $\boldsymbol{f}^{s}\left(\boldsymbol{t}^{s}\right)$ (dashed red), T-waves with only amplitude variability (we assumed $\boldsymbol{\gamma}^{*}\left(\boldsymbol{t}^{r}\right)=\boldsymbol{t}^{r}$ in this example for clarity). Panel (b.2) shows the remaining non-linear amplitude variability after normalization.
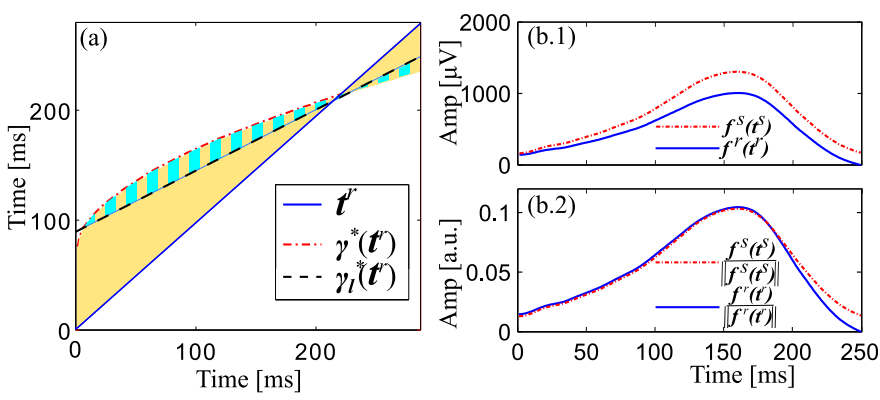

Fig. 2. Calculation of the non-linear warping and amplitude information. Panel (a): The area between $\gamma^{*}\left(\boldsymbol{t}^{r}\right)$ and $\boldsymbol{t}^{r}$ (yellow region) represents the total warping information, quantified by $d_{w}$, while the area between $\boldsymbol{\gamma}^{*}\left(\boldsymbol{t}^{r}\right)$ and $\gamma_{l}^{*}\left(\boldsymbol{t}^{r}\right)$ represents the non-linear warping information (dashed cyan region), quantified by $d_{w}^{\mathrm{NL}}$. Panel (b.1): Reference, $\boldsymbol{f}^{r}\left(\boldsymbol{t}^{r}\right)$ (solid blue), and studied, $\boldsymbol{f}^{s}\left(\boldsymbol{t}^{s}\right)$ (dashed red), T-waves with only amplitude variability $\left(\boldsymbol{\gamma}^{*}\left(\boldsymbol{t}^{r}\right)=\boldsymbol{t}^{r}\right)$. Panel (b.2): Normalized reference, $\frac{\boldsymbol{f}^{r}\left(\boldsymbol{t}^{r}\right)}{\left\|\boldsymbol{f}^{r}\left(\boldsymbol{t}^{r}\right)\right\|}$, and studied, $\frac{\boldsymbol{f}^{s}\left(\boldsymbol{\gamma}^{*}\left(\boldsymbol{t}^{r}\right)\right)}{\left\|\boldsymbol{f}^{s}\left(\boldsymbol{\gamma}^{*}\left(\boldsymbol{t}^{r}\right)\right)\right\|}$, T-
waves.

\section{Signal Preprocessing and T-wave extraction}

Preprocessing of the ECG signals included low-pass filtering at $40 \mathrm{~Hz}$ with a butterworth filter of order 6 to remove electric and muscle noise but still allow QRS detection, cubic splines interpolation for baseline wander removal and ectopic beats detection.

A single-lead-plus-rules delineation technique [29] was applied over all leads in the ECG record to delimitate the Twaves. Then, principal component analisys was calculated lead-wise over the selected T-waves to emphasize the T-wave components, improve its delineation and enhance morphological differences [30]. Finally, the first principal component was further delineated using a single-lead technique [31], and the $\mathrm{T}$-waves were confined from the $\mathrm{T}$-wave onset and $\mathrm{T}$-wave end delineation marks. Then, each T-wave was further lowpass filtered at $20 \mathrm{~Hz}$, using a butterworth filter of order 6 , to remove remaining out-of-band high frequency components 
that could potentially corrupt the T-wave shape. The filtered Twaves were visually checked for artifacts related to onset/end filtering discontinuities, observing no issues in this respect.

Before applying the warping algorithm, the reference and the studied T-waves were aligned according to their gravity centers, so that $\gamma^{*}\left(\boldsymbol{t}^{r}\right)$ is only dependent on changes in the T-wave morphology, and not on global shifts. The center of gravity was calculated as the shift in $\boldsymbol{t}^{s}$ that offered the maximum cross-correlation between $\boldsymbol{f}^{r}$ and $\boldsymbol{f}^{s}$. In case $\boldsymbol{f}^{r}$ and $\boldsymbol{f}^{s}$ had different polarities, $\boldsymbol{f}^{s}$ was inverted to match the polarity of $\boldsymbol{f}^{r}$.

\section{DATA SETS}

\section{A. Simulated Variability in a Computer-Generated ECG}

The accuracy of $d_{w}, d_{a}, d_{w}^{\mathrm{NL}}$ and $d_{a}^{\mathrm{NL}}$ in capturing linear and non-linear T-wave time and amplitude variations was assessed by simulating controlled variations in the T-wave duration and amplitude under the presence of different levels of additive noise.

Let the T-wave from a reference noise-free cardiac beat, sampled at $1 \mathrm{kHz}$, be the reference T-wave, $\boldsymbol{f}^{r}\left(\boldsymbol{t}^{r}\right)$. This reference cardiac beat was obtained from the first principal component, calculated as described in section II-C, over the 8 -standard leads recorded at supine position from a healthy subject.

The non-linear T-wave amplitude variability was modelled by adding a sinusoidal wave, of period 0.25 times $N_{r}$ and amplitude function of each beat, to $\boldsymbol{f}^{r}\left(\boldsymbol{t}^{r}\right)$ in the following way:

$$
\begin{aligned}
\boldsymbol{f}_{i}^{\mathrm{NL}}\left(\boldsymbol{t}^{r}\right) & =\boldsymbol{f}^{r}\left(\boldsymbol{t}^{r}\right)+c(i) \cdot \sin \left(2 \pi \frac{1}{4 N_{r}} \boldsymbol{t}^{r}\right), \\
c(i) & =150 \cdot \sin \left(\frac{\pi\left(\frac{I}{2}+i-1\right)}{I}\right), \quad i=1, \ldots, I,
\end{aligned}
$$

where $i$ is the heart beat index. Then, the T-wave linear amplitude variability was modelled by multiplying the deviations from the iso-electric line of $\boldsymbol{f}_{i}^{\mathrm{NL}}\left(\boldsymbol{t}^{r}\right)$ by a factor sinusoidally modulated across beats:

$$
\boldsymbol{f}_{i}^{s}\left(\boldsymbol{t}^{r}\right)=\boldsymbol{f}_{i}^{\mathrm{NL}}\left(\boldsymbol{t}^{r}\right) \cdot\left(1+0.15 \cdot \sin \left(\frac{\pi\left(\frac{I}{2}+i-1\right)}{I}\right)\right)
$$

Next, linear variations in the duration of the T-wave were simulated according to:

$$
\boldsymbol{t}_{i}^{l}=\gamma_{i}\left(\boldsymbol{t}^{r}\right), \quad i=1, \ldots, I
$$

where now $\gamma_{i}\left(\boldsymbol{t}^{r}\right)$ is the operator that up- and downsamples $\boldsymbol{t}^{r}$ according to the sampling factor, $\alpha(i)$ :

$$
\alpha(i)=\frac{0.6 \cdot(i-1)}{(I-1)}+0.7, \quad i=1, \ldots, I
$$

when simulating large time variations (like those found under appreciably different $R R$ values, which for a reference $R R=$ $1 \mathrm{~s}$, would produce an $R R$ spanning from $R R=0.7 \mathrm{~s}(85$ bpm) to $R R=1.3 \mathrm{~s} \mathrm{(46} \mathrm{bpm),} \mathrm{representing} \mathrm{a} \mathrm{heart} \mathrm{rhythm}$ change after moving from activity to relax, as an example, [32]) and:

$$
\alpha(i)=\frac{0.2 \cdot(i-1)}{(I-1)}+0.9, \quad i=1, \ldots, I
$$

when simulating small time variations (like those found when analizing short-term variability, which for a reference $R R=1$ $\mathrm{s}$, would produce an $R R$ spanning from $R R=0.9 \mathrm{~s}(66 \mathrm{bpm})$ to $R R=1.1 \mathrm{~s}(54 \mathrm{bpm})$, representing the beat-to-beat heart rate variability under stationary conditions [32]).

Non-linear variations in the temporal domain of the T-wave were introduced by adding a sinusoidal modulation of period $N_{r}$ and linearly varying amplitude, guaranteeing a monotonic increasing function:

$$
\begin{aligned}
\boldsymbol{t}_{i}^{s} & =\boldsymbol{t}_{i}^{l}+d(i) \frac{N_{r}}{N_{s_{i}}} \cdot \sin \left(2 \pi \frac{1}{N_{r}} \boldsymbol{t}_{i}^{l}\right), \\
d(i) & =\frac{30(i-1)}{I-1}-15, \quad i=1, \ldots, I
\end{aligned}
$$

where $N_{s_{i}}$ is, now, the duration of $\boldsymbol{t}_{i}^{l}$.

The re-parameterized $i$-th T-wave was, then, obtained using:

$$
\boldsymbol{f}_{i}^{s}\left(\boldsymbol{t}_{i}^{s}\right)=\boldsymbol{f}_{i}^{s}\left(\boldsymbol{\gamma}_{i}\left(\boldsymbol{t}^{r}\right)+d(i) \frac{N_{r}}{N_{s_{i}}} \cdot \sin \left(2 \pi \frac{1}{N_{r}} \boldsymbol{\gamma}_{i}\left(\boldsymbol{t}^{r}\right)\right)\right) \text {. }
$$

The $i$-th modulated cardiac beat was obtained by transforming $\boldsymbol{f}^{r}\left(\boldsymbol{t}^{r}\right)$ to $\boldsymbol{f}_{i}^{s}\left(\boldsymbol{t}_{i}^{s}\right)$. A simulated ECG signal was obtained by concatenating the $I=300$ modulated cardiac beats. This led to a 300-beat ECG signal which was filtered as explained in section II-C, and the T-waves were selected using the known delimitation marks. The morphological average of the $300 \mathrm{~T}$ waves, $\overline{\boldsymbol{f}}\left(\boldsymbol{t}^{r}\right)$, was obtained with the algorithm explained in Appendix B, and this mean warped T-wave was chosen as the reference for comparison.

The reference $\boldsymbol{d}_{w_{r}}^{r}=\left[d_{w}^{r}(1), \ldots, d_{w}^{r}(I)\right], \quad \boldsymbol{d}_{a}^{r}=$ $\left[d_{a}^{r}(1), \ldots, d_{a}^{r}(I)\right], \quad d_{w}^{\mathrm{N} \mathrm{N}^{r}}=\left[d_{w}^{\mathrm{NL}^{r}}(1), \ldots, d_{w}^{\mathrm{NL}^{r}}(I)\right]$ and $\boldsymbol{d}_{a}^{\mathrm{NL}}=\left[d_{a}^{\mathrm{NL}^{r}}(1), \ldots, d_{a}^{\mathrm{NL}^{r}}(I)\right]$ series were obtained by comparing each $\boldsymbol{f}_{i}^{s}\left(\boldsymbol{t}_{i}^{s}\right)$ with $\boldsymbol{f}\left(\boldsymbol{t}^{r}\right)$, following the procedure explained in section II-A.

Then, zero mean Laplacian noise was iteratively added to the simulated ECG signal, such that the signal-tonoise ratio $(S N R)$ was, in decibels $(\mathrm{dB}): S N R=$ $\{5,10, \ldots, 35\}$. The estimated $\underset{\mathrm{SNR}}{\boldsymbol{S}_{w}^{\mathrm{SNR}}}=\left[d_{w}^{\mathrm{SNR}}(1), \ldots, d_{w}^{\mathrm{SNR}}(I)\right]$, $\boldsymbol{d}_{a}^{\mathrm{SNR}}=\left[d_{a}^{\mathrm{SNR}}(1), \ldots, d_{a}^{\mathrm{SNR}}(I)\right], \boldsymbol{d}_{w}^{\mathrm{NL}}{ }^{\mathrm{SNR}}=\left[d_{w}^{\mathrm{NL}}(1), \ldots, d_{w}^{\mathrm{NL}}{ }^{\mathrm{SNR}}(I)\right]$ and $\boldsymbol{d}_{a}^{\mathrm{NL}}=\left[d_{a}^{\mathrm{NL}}{ }^{\mathrm{SNR}}(1), \ldots, d_{a}^{\mathrm{NL}}{ }^{\mathrm{SNR}}(I)\right]$ series were obtained by comparing the T-waves from the noisy modulated ECG signal, after preprocessing as explained in II-C, with their mean warped T-wave. The normalized relative errors were, then, calculated as:

$$
e_{d}(S N R)=\sqrt{\frac{\sum_{i=1}^{I}\left(d^{\mathrm{SNR}}(i)-d^{r}(i)\right)^{2}}{\sum_{i=1}^{I}\left(d^{r}(i)\right)^{2}}} \times 100
$$

where $d=\left\{d_{w}, d_{a}, d_{w}^{\mathrm{NL}}, d_{a}^{\mathrm{NL}}\right\}$. The noise generation and relative error measurement steps were repeated 50 times to have robust relative error values.

\section{B. Simulated Variability in an Electro-Physiological Model}

The relation between changes in myocardial repolarization dynamics and $d_{w}, d_{a}, d_{w}^{\mathrm{NL}}$, and $d_{a}^{\mathrm{NL}}$ was explored using an electro-physiological model [33], as described in Appendix C. This model presents a formalization of the forward problem in which action potentials at $M$ ventricular sites are projected onto the body surface. The action potentials repolarization 
time at each cardiac site $m$ is given by $\rho_{m}=\bar{\rho}+\Delta \rho_{m}$, where $\bar{\rho}$ is the mean repolarization time and $\Delta \rho_{m}$ represents the deviation of the local repolarization time at cardiac site $m$ from $\bar{\rho}$. The standard deviation of $\Delta \rho_{m}, \sigma$, is a measure of the global dispersion of repolarization. Ventricular action potential data was obtained from a normal male, and the ECG leads were calculated as described in Appendix C. Next, principal component analysis was performed over the ECG leads and the first principal component was preprocessed and delineated as explained in section II-C. The extracted T-wave was considered as the reference $\mathrm{T}$-wave in this simulation study.

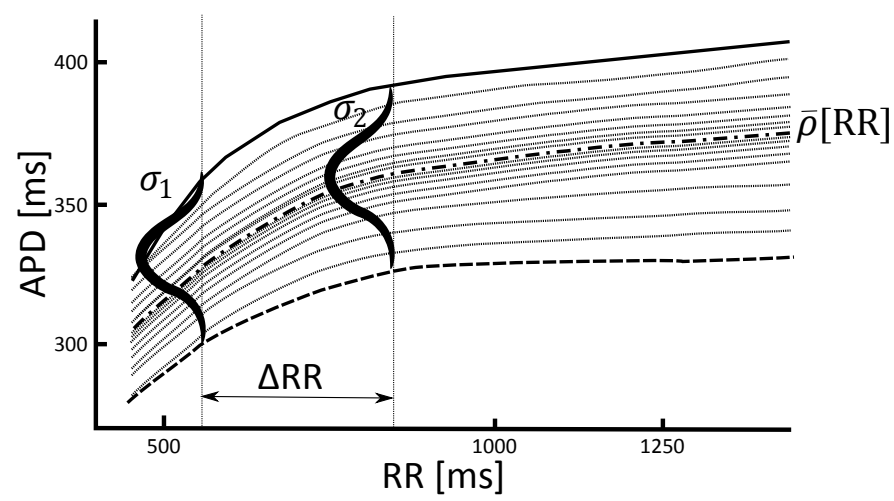

Fig. 3. Dynamic restitution curves between different ventricular regions. $\sigma$ indicates the standard deviation of the repolarization times dispersion at each value of $R R$ interval.

We assessed the morphological variability reflected on the $j$-th T-wave using $d_{w}, d_{a}, d_{w}^{\mathrm{NL}}$ and $d_{a}^{\mathrm{NL}}$, where $j=1, \ldots, 5$ is the level of variation in $\rho_{m}$ at each situation, under four scenarios:

1) Lengthening of the mean repolarization time, $\bar{\rho}$, according to the following equation:

$$
\bar{\rho}(j)=\bar{\rho}+25 \cdot(j-1), \quad j=1, \ldots, 5
$$

where $\bar{\rho}(j)$ is in ms. This is equivalent to move from a beat with $\sigma_{1}$ to other beat with $\sigma_{2}=\sigma_{1}$ and different $\bar{\rho}$, if $\bar{\rho}$ is varying with the $R R$ interval (see Figure 3 ).

2) Increasing the repolarization time dispersion, $\sigma$, using the following equation:

$$
\begin{aligned}
\Delta \rho_{m}(j) & =\Delta \rho_{m}(1+0.2(j-1)), \quad j=1, \ldots, 5 \\
\sigma(j) & =\sqrt{\frac{1}{M-1} \sum_{m=1}^{M}\left(\Delta \rho_{m}(j)-\bar{\rho}\right)^{2}}
\end{aligned}
$$

where $\Delta \rho_{m}(j)$ and $\sigma(j)$ are in ms. This is equivalent to move from a beat with $\sigma_{1}$ to a beat with $\sigma_{2} \neq \sigma_{1}$ and same $\bar{\rho}$ (see Figure 3 ).

3) In the third scenario we combined simulations 1 and 2.

4) Progressive variations of the epicardial action potential durations (APDs), defined as the difference between the maximum repolarization and depolarization slopes, (by only modifying the repolarization times, $\rho_{m}$, of epicardial nodes) towards the mean endocardial APD. This emulates the reduction in the dispersion of repolarization after reducing the spatial heterogeneity of $I_{k s}$ current as reported in [10].

Principal component analysis was performed on the resulting ECG leads, and the first principal component was preprocessed and delineated as explained in section II-C. The subsequent $d_{w}(j), d_{a}(j), d_{w}^{\mathrm{NL}}(j)$, and $d_{a}^{\mathrm{NL}}(j)$ series were obtained for each scenario by comparing the resulting $j$-th $\mathrm{T}$-wave with the reference $\mathrm{T}$-wave.

\section{Real ECG}

In this section, we focused the ECG study on the tilt table test since it is a standard procedure that induces variations in the modulation of the autonomic nervous system, i.e. heart rate, and in the repolarization features, i.e. T-wave morphology [16]. Therefore, our objective was to evaluate the ability of $d_{w}$, $d_{a}, d_{w}^{\mathrm{NL}}$ and $d_{a}^{\mathrm{NL}}$ to capture the T-wave morphological changes induced by the orthostatic stress, and their interaction with changes in the heart rate. ECG recordings from 17 healthy subjects (age $28.5 \pm 2.8$ years, 11 males) with no previous medical history related to cardiovascular diseases, acquired at the University of Zaragoza (ANS-UZ database) were analysed [16]. Each recording consisted of 8 ECG leads, sampled at 1 $\mathrm{KHz}$, acquired during a 13-min head-up tilt test (4-min supine, 5-min at $70^{\circ}, 4$-min supine). Each 8-lead ECG signal was preprocessed and the T-waves from every beat were delimited as explained in section II-C.

We assumed stationariness in three windows, early supine (ES), Tilt (TL), and late supine (LS), as done in [34]. These windows had a length of 20 beats and finished $30 \mathrm{~s}$ before any transition during the tilt test. Then, for each subject and window, we calculated the $R R, Q T, T W$, and $T_{\text {pe }}$ interval values as the differences between subsequent QRS fiducial points, T-wave end and QRS complex onset, T-wave end and T-wave onset, and T-wave end and T-wave peak, respectively, from the annotation marks calculated in section II-C [31]. Similarly, the $T A$ values were obtained from the T-wave amplitude values. Finally, for each subject and window, we calculated the median $R R, Q T, T W, T_{\mathrm{pe}}$ and $T A$ values. In addition, the mean warped T-wave of the $20 \mathrm{~T}$-waves in each window and subject was calculated following the algorithm explained in Appendix B.

1) Analysis of long-term T-wave morphological variability: To evaluate the variations of the T-wave produced by the orthostatic stress, we compared each mean warped T-wave with the other two $((L S-E S),(T L-E S)$ and $(L S-T L))$, and we calculated the difference between each median $R R, T_{\mathrm{pe}}, T W$, $Q T$ and $T A$ values and the other two, obtaining three values of $d_{w}, d_{a}, d_{w}^{\mathrm{NL}}, d_{a}^{\mathrm{NL}}, \Delta R R, \Delta T_{\mathrm{pe}}, \Delta T W, \Delta Q T$ and $\Delta T A$ per subject.

2) Analysis of short-term T-wave morphological variability: To evaluate the variability of the T-wave at steady-state conditions, we calculated the median absolute deviation of $Q T$, $T W, T_{\mathrm{pe}}$ and $T A$ with respect to their median value, for each subject and window [35]. Regarding the proposed morphological markers, we calculated the median of the 20 absolute values of $d_{w}, d_{a}, d_{w}^{\mathrm{NL}}$ and $d_{a}^{\mathrm{NL}}$, obtained when comparing each T-wave in the window with its mean warped T-wave. Finally, we measured the $S N R$ value of the T-waves in the window, defined as the ratio between the root mean squared values of their arithmetic mean T-wave and the difference between each $\mathrm{T}$-wave and this arithmetic mean $\mathrm{T}$-wave, in decibels. 
Therefore, we assumed that noise level was higher than the variability itself. This definition can be argued at situations where the T-wave variability is high, as compared to noise. However, for Holter ECG records at stationary conditions, it is expected to have low T-wave variability and high noise levels. Then, for general cases, this will be an appropriate assumption when estimating correlation for the whole range of SNR.

3) Statistical analysis: The Spearman's correlation coefficient was used to quantify the strength of the linear correlation. The Wilcoxon signed-rank test was used in pairwise comparisons. A $p$ value of $\leq 0.05$ was considered as statistically significant. On each boxplot, the central mark is the median, the edges of the box are the 25th and 75th percentiles, and the whiskers extend to the most extreme data points not considered as outliers. The notches represent the $95 \%$ confidence interval of the median, calculated as $q 2 \pm 1.57(q 3-q 1) / \sqrt{n}$, where $q 2$ is the median, $q 1$ and $q 3$ are the 25th and 75th percentiles, respectively, and $n$ is the number of subjects. Therefore, since the sample is small (17 subjects), the notches might extent beyond the end of the box in some cases. Computations were executed using Matlab 7.10.0 (2010a), Intel Core i7-2600 CPU, 3.40GHz, 8.00 GB RAM.

\section{Results}

\section{A. Simulated Variability in a Computer-Generated ECG}

Figure 4 shows six examples of the linear and non-linear time and amplitude simulations of the T-wave. The reference T-wave, $\boldsymbol{f}^{r}\left(\boldsymbol{t}^{r}\right)$ is displayed in solid blue in every panel, and the simulated T-wave, $\boldsymbol{f}_{i}^{s}\left(\boldsymbol{t}_{i}^{s}\right)$ is plotted in dashed red, for $i=\{1,60,120,180,240,300\}$.

Figure 5 shows the evolution of $d_{w}^{r}$ (a), $d_{a}^{r}(\mathrm{~b}), d_{w}^{\mathrm{N}^{r}}$ (c), and $d_{a}^{\mathrm{N}^{r}}$ (d) series as the linear and non-linear time and amplitude modulations induced in the T-wave change. The evolution of $d_{w}^{20}, d_{a}^{20}, d_{w}^{\mathrm{NL}^{20}}$, and $d_{a}^{\mathrm{NL}^{20}}$ is illustrated in red.

Figure 6 shows the relative error between $\boldsymbol{d}_{w}^{\mathrm{SNR}}$ and $\boldsymbol{d}_{w}^{r}(\mathrm{a})$, $\boldsymbol{d}_{a}^{\mathrm{SNR}}$ and $\boldsymbol{d}_{a}^{r}(\mathrm{~b}), \boldsymbol{d}_{w}^{\mathrm{N} \mathrm{LNR}}$ and $\boldsymbol{d}_{w}^{r}(\mathrm{c})$, and $\boldsymbol{d}_{a}^{\mathrm{N}}{ }^{\mathrm{SNR}}$ and $\boldsymbol{d}_{a}^{r}(\mathrm{~d})$, for different $S N R$ when simulating large (red) and small (black) linear time variations.

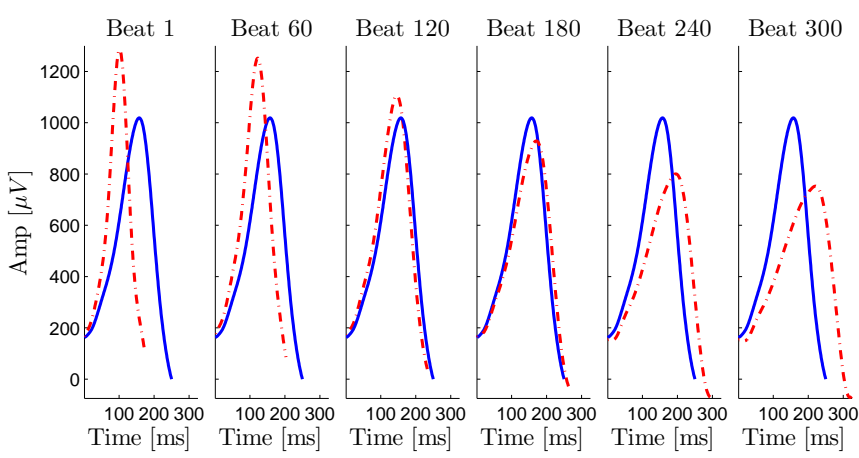

Fig. 4. Evolution of linear and non-linear time and amplitude simulated variations. The reference T-wave, $\boldsymbol{f}^{r}\left(\boldsymbol{t}^{r}\right)$, is displayed in solid blue, and the simulated T-wave, $\boldsymbol{f}_{i}^{s}\left(\boldsymbol{t}_{i}^{s}\right)$, is plotted in dashed red, for $i=$ $\{1,60,120,180,240,300\}$.
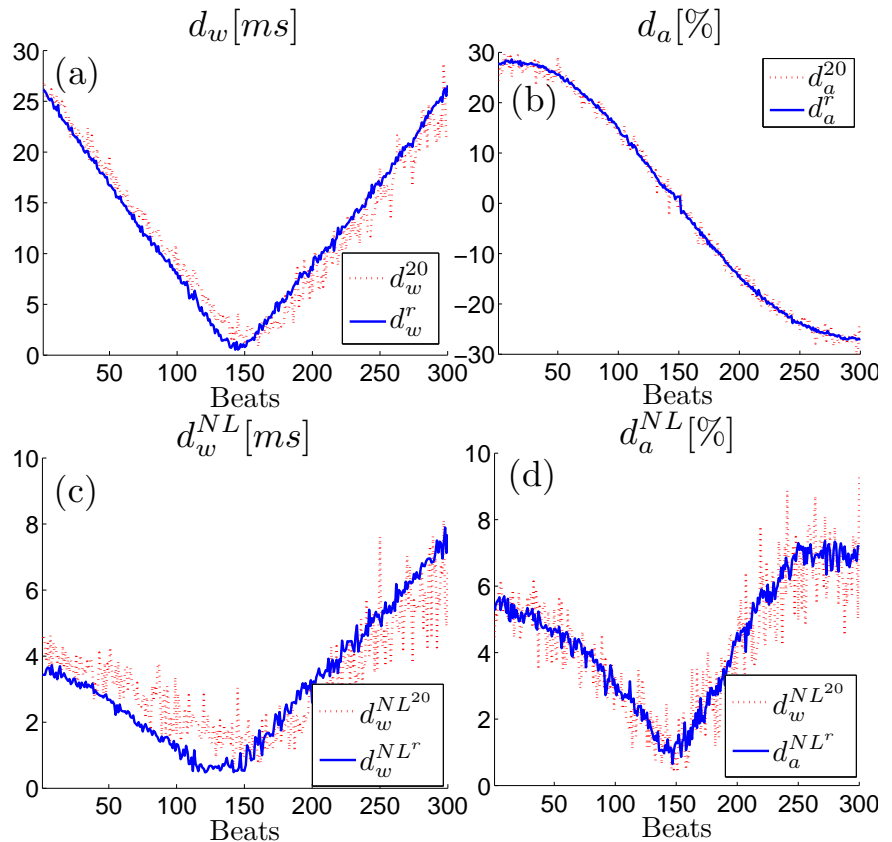

Fig. 5. Evolution of $d_{w}, d_{a}, d_{w}^{\mathrm{NL}}$ and $d_{a}^{\mathrm{NL}}$ with respect to the level of linear and non-linear time and amplitude modulations in a noise-free scenario (solid blue) and under the presence of additive Laplacian noise with $S N R=20 \mathrm{~dB}$ (dashed red), when simulating large temporal variations, as in eq.(11).

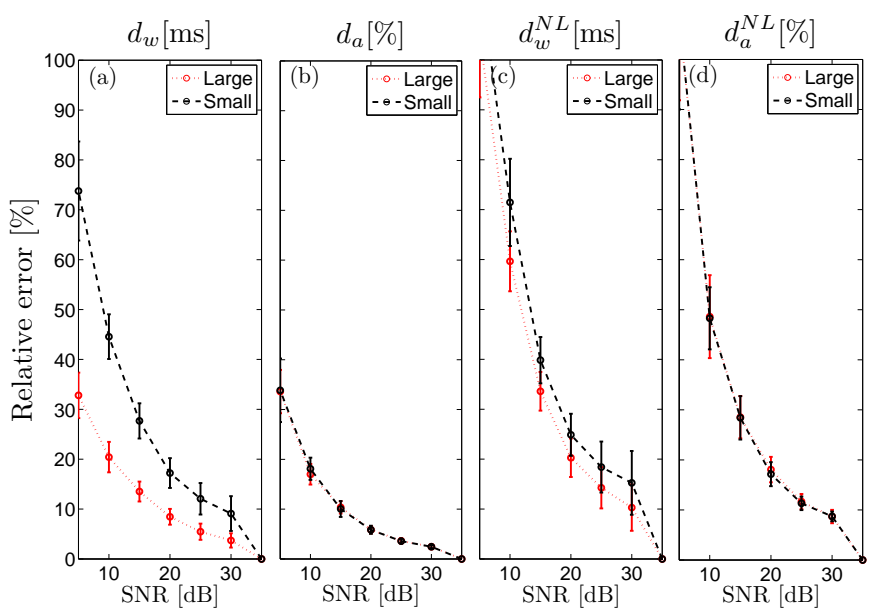

Fig. 6. Relative error between reference and estimated $d_{w}, d_{a}, d_{w}^{\mathrm{NL}}$ and $d_{a}^{\mathrm{NL}}$ under the presence of additive Laplacian noise, when simulating large (solid) and small (dotted) time variations, as in eq.(11) and (12), respectively. Nonlinear time variations and linear and non-linear amplitude changes are the same in all situations.

\section{B. Simulated Variability in an Electro-Physiological Model}

Figure 7, panels (a), show the simulation of the first scenario described in section III-B, i.e. lengthening of the mean repolarization time, $\bar{\rho}$. Panels (b) illustrate the simulation of the second scenario, i.e. an increment of the dispersion of action potential repolarization times, $\sigma$. Panels (c) show the combined simulation of $\bar{\rho}$ and $\sigma$ variation. Panels (d) show the simulation results of the fourth scenario described in section III-B. Panels (a.1), (a.2) and (a.3) illustrate the range between the minimum and the maximum APD, with respect to $\bar{\rho}$ (a.1), $\sigma$ (b.1) and $\bar{\rho}$ (c.1). Panel (d.1) shows an endocardial action potential, which does not change. Panels (a.2), (b.2) and (c.2) illustrate 

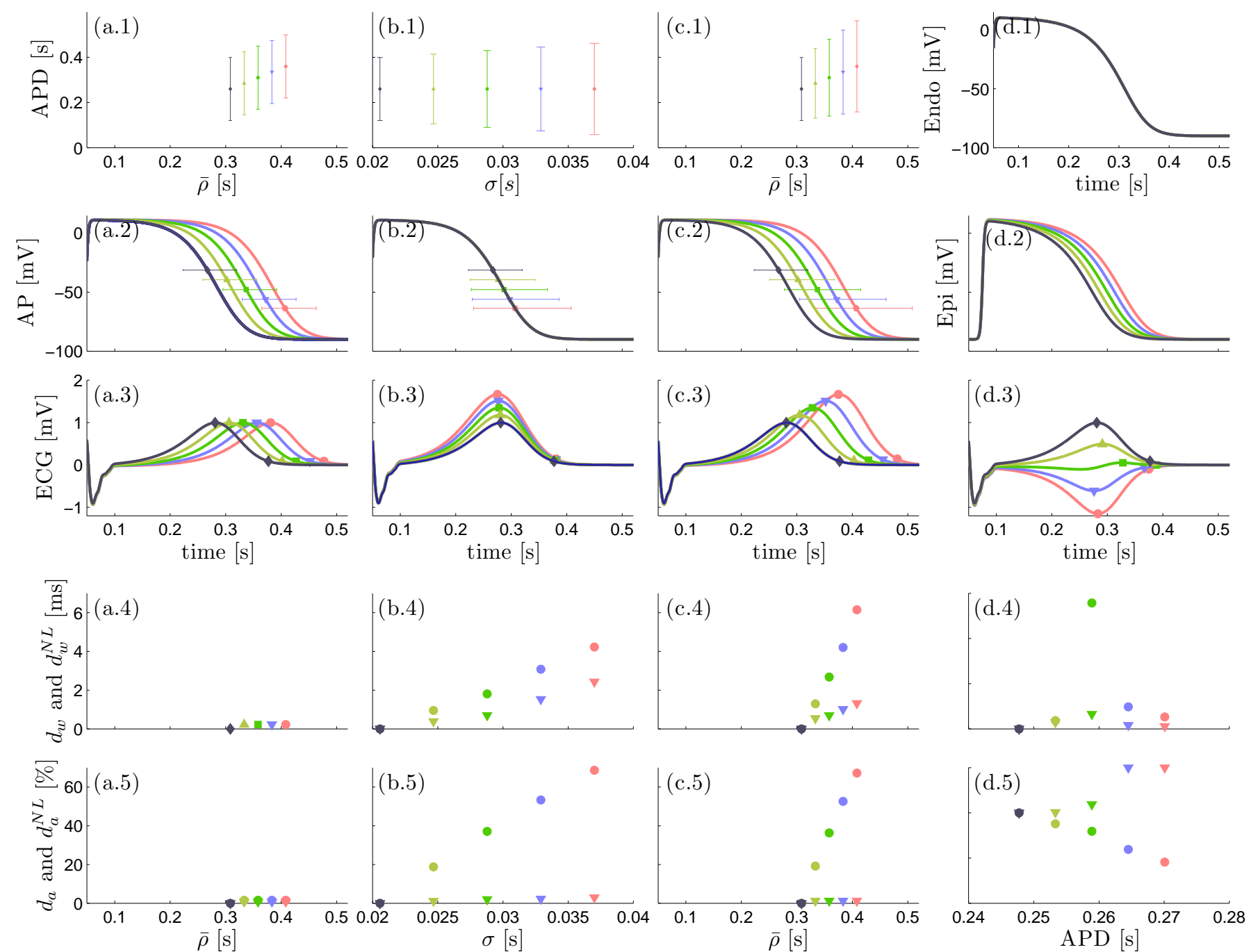

Fig. 7. Performance evaluation using an electro-physiological cardiac model. Panels (a.1-5), increments of the mean repolarization time, $\bar{\rho}$. Panels (b.15), increments of the standard deviation of the repolarization time dispersion, $\sigma$. Panels (c.1-5), combined variation of $\bar{\rho}$ and $\sigma$. Panels (d.1-5), progressive variations of the epicardial APDs. (a.1-c.1): range between the minimum and the maximum APD with respect to the variation. (a.2-c.2): mean action potentials with respect to the variation. (d.1-2): Simulated action potential of endocardial and epicardial cells, respectively. (a.3-d.3): ECG beats corresponding to the modified action potentials. (d.4-d.5): $d_{w}$ and $d_{a}$ values (circles) and $d_{w}^{\mathrm{NL}}$ and $d_{a}^{\mathrm{NL}}$ values (triangles). Each color indicates a level of variation. Horizontal bars represent the range between the minimum and the maximum repolarization time.

the mean action potential. The horizontal bars represent the corresponding range between the minimum and the maximum repolarization time. It should be noted that they are located slightly away from the mean repolarization time to avoid overlapping with the neighbouring horizontal bars, but it is only a matter of display. Panel (d.2) shows an epicardial action potential and the result of progressively increasing its duration (each color represents a different duration). Panels (a.3-d.3) show the principal component as a result of the projection of the modified action potentials. Panels (a.4-d.5) show the values of $d_{w}$ (circle) and $d_{w}^{\mathrm{NL}}$ (triangle), and of $d_{a}$ (circle) and $d_{a}^{\mathrm{NL}}$ (triangle), respectively in the four simulations.

\section{Real ECG}

1) Analysis of long-term T-wave morphological variability: Figure 8, left column, shows the boxplots of $\Delta R R$ (a.1), $\Delta Q T, \Delta \mathrm{TW}$ and $\Delta T_{\mathrm{pe}}(\mathrm{a} .2), \Delta T A$ (a.3), $d_{w}$ and $d_{w}^{\mathrm{NL}}$ (a.4) and $d_{a}$ and $d_{a}^{\mathrm{NL}}$ (a.5) values, measured for (LS-ES), (TL-ES) and $(L S-T L)$.

Table I shows the correlation coefficient, when comparing $L S-E S, T L-E S$, and $L S-T L$, between (i) $d_{w}$ and $d_{a}$, and between $d_{w}^{\mathrm{NL}}$ and $d_{a}^{\mathrm{NL}}$; (ii) $d_{w}$ and $d_{w}^{\mathrm{NL}}$ and each of the repolarization markers measuring time intervals, $\Delta Q T, \Delta T W$ and $\Delta T_{\mathrm{pe}}$; (iii) $d_{a}$ and $d_{a}^{\mathrm{NL}}$ and the repolarization marker measuring amplitude differences, $\Delta T A$; and (iv) $d_{w}, d_{w}^{\mathrm{NL}}, d_{a}$ and $d_{a}^{\mathrm{NL}}$ and $\Delta R R$.

2) Analysis of short-term T-wave morphological variability: Figure 8, right column, shows the boxplots of the mean values of $R R$ (b.1), median absolute deviation values of $Q T, T W$ and $T_{\mathrm{pe}}$ (b.2) and $T A$ (b.3) with respect to their median value, $d_{w}$ and $d_{w}^{\mathrm{NL}}$ (b.4) and $d_{a}$ and $d_{a}^{\mathrm{NL}}$ (b.5) at the three windows.

Table II shows the correlation coefficient between $d_{w}, d_{w}^{\mathrm{NL}}$, $d_{a}$ and $d_{a}^{\mathrm{NL}}$, and the median $R R$ and $S N R$ values for each window separately and when pooling them into a single group $E S+T L+L S$. 

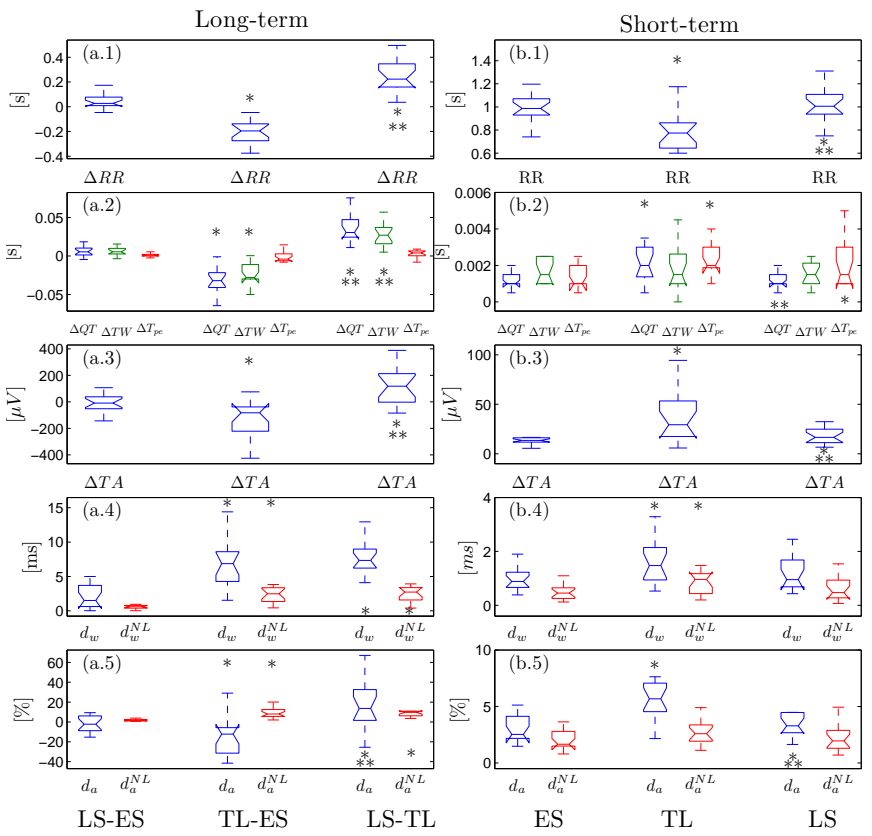

Fig. 8. Left column, boxplots of $\Delta R R$ (a.1), $\Delta Q T, \Delta \mathrm{TW}$ and $\Delta T_{\mathrm{pe}}$ (a.2), $\Delta T A$ (a.3), $d_{w}$ and $d_{w}^{\mathrm{NL}}$ (a.4) and $d_{a}$ and $d_{a}^{\mathrm{NL}}$ (a.5) values, across subjects, between the three windows along the recording. Right column, boxplots of the mean values of $R R$ (b.1), median absolute deviation of $Q T, T W$ and $T_{\mathrm{pe}}$ (b.2) and $T A$ (b.3), and mean $d_{w}$ and $d_{w}^{\mathrm{NL}}$ (b.4) and $d_{a}$ and $d_{a}^{\mathrm{NL}}$ (b.5) values at the three windows. $*$ indicates statistically significant differences with respect to the leftmost boxplot. $* *$ indicates statistically significant differences between the central and rightmost boxplots. It should be noted that left and right panels have different scale.

\begin{tabular}{lccc}
\hline \hline & & & \\
$r$ & $L S-E S$ & $T L-E S$ & $L S-T L$ \\
\hline$d_{w}$ vs. $d_{a}$ & $\mathbf{- 0 . 5 3}$ & -0.07 & 0.23 \\
$d_{w}^{\mathrm{NL}}$ vs. $d_{a}^{\mathrm{NL}}$ & $\mathbf{0 . 7 2}$ & $\mathbf{0 . 7 7}$ & $\mathbf{0 . 6 9}$ \\
$d_{w}$ vs. $\Delta Q T$ & $\mathbf{0 . 5 5}$ & -0.38 & $\mathbf{0 . 6 9}$ \\
$d_{w}^{\mathrm{NL}}$ vs. $\Delta Q T$ & 0.36 & -0.36 & 0.18 \\
$d_{w}$ vs. $\Delta T W$ & $\mathbf{0 . 8 1}$ & $\mathbf{- 0 . 6 0}$ & $\mathbf{0 . 7 1}$ \\
$d_{w}^{\mathrm{NL}}$ vs. $\Delta T W$ & $\mathbf{0 . 7 0}$ & -0.34 & 0.44 \\
$d_{w}$ vs. $\Delta T_{\mathrm{pe}}$ & $\mathbf{0 . 6 5}$ & 0.10 & -0.16 \\
$d_{w}^{\mathrm{NL}}$ vs. $\Delta T_{\mathrm{pe}}$ & 0.45 & $\mathbf{0 . 7 5}$ & $\mathbf{- 0 . 6 0}$ \\
$d_{a}$ vs. $\Delta T A$ & $\mathbf{0 . 9 5}$ & $\mathbf{0 . 8 7}$ & $\mathbf{0 . 8 8}$ \\
$d_{a}^{\mathrm{NL}}$ vs. $\Delta T A$ & -0.41 & -0.43 & 0.28 \\
$d_{w}$ vs. $\Delta R R$ & $\mathbf{0 . 5 5}$ & -0.41 & $\mathbf{0 . 6 3}$ \\
$d_{w}^{\mathrm{NL}}$ vs. $\Delta R R$ & 0.54 & -0.45 & 0.26 \\
$d_{a}$ vs. $\Delta R R$ & -0.07 & $\mathbf{0 . 5 5}$ & 0.28 \\
$d_{a}^{\mathrm{NL}}$ vs. $\Delta R R$ & 0.36 & -0.40 & 0.44 \\
\hline \hline
\end{tabular}

TABLE I

SPEARMAN'S CORRELATION COEFFICIENT, $r$, IN THE LONG-TERM ANALYSIS. SIGNIFICANT CORRELATIONS ARE INDICATED IN BOLD.

\section{Computational time}

As a reference for computational time estimation, the calculation of $d_{w}$ and $d_{a}$ when comparing two T-waves took 1.31 seconds with the personal computer and software described at the end of section II. The computation of the mean warped T-wave was slower, needing 47 seconds to calculate the mean warped T-wave of a set of $20 \mathrm{~T}$-waves. It should be advised that the algorithm has not been optimized in terms of computational time, since the current time is not considered a bottle neck for the technology to be used in clinical practice.

\begin{tabular}{lcccc}
\hline \hline & $E S$ & $T L$ & $L S$ & $E S+T L+L S$ \\
\hline & & & & \\
$d_{w}$ vs. $S N R$ & $\mathbf{- 0 . 6 8}$ & $\mathbf{- 0 . 6 0}$ & $\mathbf{- 0 . 6 1}$ & $\mathbf{- 0 . 7 0}$ \\
$d_{w}^{\mathrm{NL}}$ vs. $S N R$ & $\mathbf{- 0 . 6 1}$ & $\mathbf{- 0 . 5 5}$ & $\mathbf{- 0 . 8 1}$ & $\mathbf{- 0 . 7 4}$ \\
$d_{w}$ vs. $R R$ & -0.16 & $\mathbf{- 0 . 5 4}$ & -0.14 & $\mathbf{- 0 . 3 7}$ \\
$d_{w}^{\mathrm{NL}}$ vs. $R R$ & 0.01 & -0.40 & -0.01 & $\mathbf{- 0 . 3 0}$ \\
$d_{a}$ vs. $S N R$ & $\mathbf{- 0 . 9 3}$ & $\mathbf{- 0 . 7 1}$ & $\mathbf{- 0 . 8 3}$ & $\mathbf{- 0 . 8 5}$ \\
$d_{a}^{\mathrm{NL}}$ vs. $S N R$ & $\mathbf{- 0 . 9 0}$ & $\mathbf{- 0 . 6 9}$ & $\mathbf{- 0 . 8 6}$ & $\mathbf{- 0 . 8 4}$ \\
$d_{a}$ vs. $R R$ & -0.17 & -0.17 & -0.09 & $\mathbf{- 0 . 3 9}$ \\
$d_{a}^{\mathrm{NL}}$ vs. $R R$ & -0.19 & -0.41 & -0.28 & $\mathbf{- 0 . 4 1}$ \\
\hline \hline
\end{tabular}

TABLE II

SPEARMAN'S CORRELATION COEFFICIENT, $r$, IN THE SHORT-TERM ANALYSIS. SIGNIFICANT CORRELATIONS ARE INDICATED IN BOLD.

\section{DISCUSSION}

In this study, we introduced four novel ECG-derived indices, $d_{w}$ and $d_{a}$, that independently quantify T-wave morphological variability in the temporal and amplitude domain, respectively, and $d_{w}^{\mathrm{NL}}$ and $d_{a}^{\mathrm{NL}}$ that isolate the non-linear information present within $d_{w}$ and $d_{a}$, respectively. We evaluated the accuracy of $d_{w}, d_{a}, d_{w}^{\mathrm{NL}}$ and $d_{a}^{\mathrm{NL}}$ under the presence of additive Laplacian noise. We investigated the relationship between $d_{w}, d_{a}, d_{w}^{\mathrm{NL}}$ and $d_{a}^{\mathrm{NL}}$ and the induced morphological variations at cardiac cellular level and we evaluated the extent of T-wave time and amplitude changes produced by a tilt test in real ECG, and the correlation of $d_{w}, d_{a}, d_{w}^{\mathrm{NL}}$ and $d_{a}^{\mathrm{NL}}$ with the heart rate and with well-known time and amplitude-based repolarization markers.

\section{A. Simulated Variability in a Computer-Generated ECG}

The evaluation of the accuracy of $d_{w}, d_{a}, d_{w}^{\mathrm{NL}}$ and $d_{a}^{\mathrm{NL}}$ in detecting linear and non-linear modulations of the T-wave time domain and amplitude, respectively, in a simulated ECG signal under the presence of additive Laplacian noise confirmed that the four markers were able to robustly and independently capture both modes of variation, with no coupling between the different sources of variability, i.e. the evolution of $d_{w}$ and $d_{w}^{\mathrm{NL}}$ being independent from that of $d_{a}$ and $d_{a}^{\mathrm{NL}}$ (Figure 5). The values of relative error remained lower than $20 \%$ for $S N R=20 \mathrm{~dB}$, indicating that these markers can be robustly used in ambulatory Holter ECG (Figure 6). When the simulated time-domain variations were small, the relative error increased for low $S N R$ because noise dominates over time-domain variations of the T-wave.

\section{B. Simulated Variability in an Electro-Physiological Model}

The assessment of the relationship between T-wave time and amplitude variations and morphological changes at cardiac cellular level using an electro-physiological cardiac model [33] showed that a variation in the mean repolarization time, $\bar{\rho}$, provoked a shift in the T-wave but with no significant T-wave temporal or amplitude variation (Figure 7, (a.3)). Therefore, $d_{w}, d_{w}^{\mathrm{NL}}, d_{a}$ and $d_{a}^{\mathrm{NL}}$ were equal to zero for every $\bar{\rho}(j)((\mathrm{a} .4)-$ (a.5)). On the contrary, increments of the dispersion of action potential repolarization times, $\sigma$, produced an increment in the range of the APD (b.1) and in the range between the minimum and the maximum repolarization time (b.2). This led to an homogeneous increment in the amplitude and to a 
linear and non-linear increment of the width of the T-wave, which was reflected in higher values of $d_{w}, d_{w}^{\mathrm{NL}}$ and $d_{a}$, while $d_{a}^{\mathrm{NL}}$ remained zero (panels (b.3)-(b.5)). The combined variation of $\bar{\rho}$ and $\sigma$ produced values of $d_{w}, d_{w}^{\mathrm{NL}}, d_{a}$ and $d_{a}^{\mathrm{NL}}$ very similar to those of scenario 2. Progressive increments in the epicardial repolarization time provoked heterogeneous changes in the morphology of the T-wave (d.3) and eventually, when the mean epicardial repolarization time was longer than the mean endocardial repolarization time, its inversion. Panels (d.4)(d.5) illustrate that $d_{a}$ followed the increment in amplitude variability (due to the T-wave inversion), while $d_{w}$ measured the asymmetry present in the T-wave when its amplitude was close to zero. In this case, both $d_{w}^{\mathrm{NL}}$ and $d_{a}^{\mathrm{NL}}$ captured the nonlinear time and amplitude, respectively, variations induced by the simulation. These results suggest that $d_{w}, d_{w}^{\mathrm{NL}}, d_{a}$ and $d_{a}^{\mathrm{NL}}$ mainly represent changes in spatio-temporal dispersion of repolarization rather than in its mean, with $d_{w}^{\mathrm{NL}}$ and $d_{a}^{\mathrm{NL}}$ being only sensitive to the variations of spatio-temporal dispersion of repolarization times reflected as non-linear morphological changes of the T-wave.

\section{Real ECG}

The purpose of the long-term analysis was to evaluate the ability of the proposed markers to capture the T-wave morphological changes induced by the orthostatic stress, and their interaction with changes in the heart rate. We, then, assumed a similar situation to that from scenario 3) in section III-B, i.e. variations in repolarization dispersion as a result of variations in heart rate, but in actual ECG records. The orthostatic stress produced significant decrements of the $R R$, $Q T, T W$ and $T A$ values (Figure 8 ). Regarding $T_{\mathrm{pe}}, 13$ subjects reflected a shorter $T_{\mathrm{pe}}$ during $T L$, while this interval increased for the other 4 subjects. The proposed markers supported these results, showing significant increments in linear and nonlinear temporal and amplitude variations after an orthostatic stress. The correlation analysis (Table I) showed that $d_{w}$ was correlated with $\triangle R R, \Delta Q T$ and $\Delta T W$, whereas $d_{w}^{\mathrm{NL}}$ was only correlated with $\Delta T_{\mathrm{pe}}$. These results may indicate that the orthostatic stress produces a change in heart rate, inducing linear variations in the $Q T$ and $T W$ intervals, which dominate in $d_{w}$. The strong correlation between $d_{w}^{\mathrm{NL}}$ and $\Delta T_{\mathrm{pe}}$ suggests that $d_{w}^{\mathrm{NL}}$ is mainly related to variations in the dispersion of repolarization, independently from changes in heart rate. Regarding the amplitude markers, $d_{a}$ was highly correlated with $\Delta T A$, whereas $d_{a}^{\mathrm{NL}}$ was completely uncorrelated to it, therefore reflecting information not contained in $\Delta T A$.

The purpose of the short-term analysis was similar to that from scenario 2) in section III-B, i.e. evaluation of the variations in repolarization dispersion at stable heart rate. Results showed that the variability of $Q T$ and $T_{\mathrm{pe}}$ intervals, and $T A$ was significantly higher during $T L$ than $E S$ or $L S$. This was supported by the values of $d_{w}, d_{a}$ and $d_{w}^{\mathrm{NL}}$, which reflected significantly increased variability in $T L$ as compared to supine (Figure 8). There was a weak negative correlation between $d_{w}, d_{a}, d_{w}^{\mathrm{NL}}$ and $d_{a}^{\mathrm{NL}}$, and the median $R R$ (Table II), indicating that the short-term changes in dispersion of repolarization, reflected on the ECG through linear and nonlinear T-wave morphological variations, increase at shorter
$R R$ interval values. This may be in corcondance with studies reporting steeper slopes of repolarization restitution at shorter RR values (Figure 3) [6]. The correlation values with respect to the $S N R$ indicate that beat-to-beat analysis should be done on clean recordings.

Considering the results of this work and, in view of the large number of publications reporting an association between repolarization instabilities and arrhythmogenesis [3, 13, 17, $18,36]$, the proposed methodology might provide additional information that may eventually lead to an improved SCD risk prediction.

\section{Technical considerations}

Warping of time domain has often been used in the ECG signal to compensate for the missalignments before measuring amplitude differences, or to quantify time interval variabilities, like those from the QT interval, using linear [37-39] and non-linear [40] time warping. The most common algorithm for non-linear time warping is the dynamic time warping (DTW) [40], but it fails when the two observed functions also present amplitude variabilities [24]. To prevent this problem, the derivative DTW was proposed [41]. This modification aligns the derivative of the observed functions, rather than their amplitude values. The SRSF used in this work makes the norm to be minimized in equation (3) to become a proper distance and overcome the "pinching effect", as explained in [23-25]. Also, a novel technique to allow for warping in both temporal and amplitude domains has been recently proposed to enable complex adaptations to the morphology of the waveform [22]. The novelty of this work is based on the extraction of indices from the warping information and from the warped signals to quantify the amount of morphological variability.

We applied principal component analysis to obtain a lead projected onto the direction of maximal energy of the $\mathrm{T}$ wave. However, the selection of the lead does not affect the technicalities of the proposed algorithm, since this methodology can be applied to any single lead, which is its main advantage with respect to other methodologies that require the vectorcardiogram, or the information of more than one lead [19].

\section{E. Limitations of the Study}

There are several aspects that could influence the estimation reliability of the proposed indices. First, due to the high sensitivity of the warping function, erroneous extraction of the morphology of the T-wave, due to excessive noise or delineation errors, will lead to incorrect values of $d_{w}$ and $d_{w}^{\mathrm{NL}}$, and an incorrect warping. Therefore, the markers $d_{a}$ and $d_{a}^{\mathrm{NL}}$ could also be affected, coupling, in some way, the robustness and sensitivity of the indices. Second, although the proposed markers capture many T-wave morphologies, there can appear extreme morphological variations which will lead to meaningless interpretations of $d_{w}, d_{a}, d_{w}^{\mathrm{NL}}$ and $d_{a}^{\mathrm{NL}}$. However, we have not found any such extreme variation in the analysed T-waves, since even for biphasic or S-shaped T-waves we still obtained interpretable results. Next, we selected the first principal component as the lead capturing the direction of 
maximal variation of the repolarization gradient. Although we assumed that a projection of the maximal energy onto this component also implied a maximal projection of the repolarization variability, given the physiological additive generation of the ECG signals, this may not always be guaranteed. Finally, this first principal component may be dominated by precordial lead T-waves, which have the highest amplitude and, therefore, in this study, the algorithm did not capture the morphological variation reflected in other leads.

\section{CONCLUSION}

In this study, we introduce two ECG-derived markers, $d_{w}$ and $d_{a}$, and their non-linearly restricted versions, $d_{w}^{\mathrm{NL}}$ and $d_{a}^{\mathrm{NL}}$, to quantify single-lead $\mathrm{T}$-wave morphological variability by time-warping metrics. Our findings demonstrate that variations in the dispersion of repolarization, reflected in the ECG through changes in the temporal and amplitude organization of the T-wave, can be robustly captured by $d_{w}$ and $d_{a}$, respectively, with $d_{w}^{\mathrm{NL}}$ and $d_{a}^{\mathrm{NL}}$ measuring the heterogeneous T-wave variations. Future clinical situations will evaluate the strong potential of the proposed markers of T-wave time and amplitude variability to be used as arrhythmic risk predictors.

\section{APPENDIX A}

\section{Dynamic Programming Algorithm}

Let $\boldsymbol{f}^{r}\left(\boldsymbol{t}^{r}\right)=\left[f^{r}\left(t^{r}(1)\right), \ldots, f^{r}\left(t^{r}\left(N_{r}\right)\right)\right]^{T}$ and $\boldsymbol{f}^{s}\left(\boldsymbol{t}^{s}\right)=$ $\left[f^{s}\left(t^{s}(1)\right), \ldots, f^{s}\left(t^{s}\left(N_{s}\right)\right)\right]^{T}$, where $\boldsymbol{t}^{r}=\left[t^{r}(1), \ldots, t^{r}\left(N_{r}\right)\right]^{T}$ and $\boldsymbol{t}^{s}=\left[t^{s}(1), \ldots, t^{s}\left(N_{s}\right)\right]^{T}$ and $N_{r}$ and $N_{s}$ being the total duration of $\boldsymbol{t}^{r}$ and $\boldsymbol{t}^{s}$, be two given functions and we want to solve for:

$$
\boldsymbol{\gamma}^{*}\left(\boldsymbol{t}^{r}\right)=\underset{\gamma\left(\boldsymbol{t}^{r}\right)}{\arg \min }\left(\left\|\boldsymbol{f}^{r}\left(\boldsymbol{t}^{r}\right)-\boldsymbol{f}^{s}\left(\gamma\left(\boldsymbol{t}^{r}\right)\right)\right\|\right)
$$

where $\gamma\left(\boldsymbol{t}^{r}\right)$ is a function that matches each point in $\boldsymbol{f}^{s}\left(\gamma\left(\boldsymbol{t}^{r}\right)\right)$ with each point in $\boldsymbol{f}^{r}\left(\boldsymbol{t}^{r}\right)$, and $\boldsymbol{\gamma}^{*}\left(\boldsymbol{t}^{r}\right)$ is the optimal matching function. We can solve a discrete approximation of this problem using dynamic programming. To decompose the large problem into several sub-problems, we define a local cost function:

$$
E\left(\tau_{1}, \tau_{2} ; \boldsymbol{\gamma}\right)=\left\|\boldsymbol{f}^{r}(\boldsymbol{\tau})-\boldsymbol{f}^{s}(\boldsymbol{\gamma}(\boldsymbol{\tau}))\right\| \quad \tau_{1} \leq \boldsymbol{\tau} \leq \tau_{2}
$$

So that the original cost function is simply $E\left(t^{r}(1), t^{r}\left(N_{r}\right) ; \gamma\right)$. A necessary condition for applying dynamic programming to such problems is that the cost function is additive in time. Consider the $2 \mathrm{D}$ time coordinates grid formed by $\left[\boldsymbol{t}^{r}, \boldsymbol{t}^{s}\right]$, with size $N_{r} \times N_{s}$. We will conveniently view $\gamma$ as a graph in this grid from $\left[t^{r}(1), t^{s}(1)\right]$ to $\left[t^{r}\left(N_{r}\right), t^{s}\left(N_{s}\right)\right]$, such that the slope of this graph is always strictly positive. Let $\left[t^{r}(a), t^{s}(b)\right]$ be a point on the grid. Since we have a constraint on the slope of the graph, there are only certain nodes, $N_{a b}$, that are allowed to go to $\left[t^{r}(a), t^{s}(b)\right]$ :

$$
N_{a b}=\left\{\left[t^{r}\left(a^{\prime}\right), t^{s}\left(b^{\prime}\right)\right] \mid t^{r}(1) \leq t^{r}\left(a^{\prime}\right)<t^{r}(a), t^{s}(1) \leq t^{s}\left(b^{\prime}\right)<t^{s}(b)\right\}
$$

Define $L\left(a^{\prime}, b^{\prime} ; a, b\right)$ as a straight line with strictly positive slope joining the nodes $\left[t^{r}\left(a^{\prime}\right), t^{s}\left(b^{\prime}\right)\right]$ and $\left[t^{r}(a), t^{s}(b)\right]$. This sets up the local optimization problem:

$$
\left[t^{r^{*}}\left(a^{\prime}\right), t^{s^{*}}\left(b^{\prime}\right)\right]_{\left.-t^{r}\left(a^{\prime}\right), t^{s}\left(b^{\prime}\right)\right) \in N_{a b}}^{\arg \min }\left(E\left(t^{r}\left(a^{\prime}\right), t^{r}(a) ; L\left(a^{\prime}, b^{\prime} ; a, b\right)\right)\right),
$$

Let $H\left(t^{r}(a), t^{r}(b)\right)$ be the minimum cumulative cost needed to reach the point $\left[t^{r}(a), t^{s}(b)\right]$, defined as:

$$
\begin{aligned}
H\left(t^{r}(a), t^{s}(b)\right) & =E\left(t^{r^{*}}\left(a^{\prime}\right), t^{r}(a) ; L\left(a^{\prime}, b^{\prime} ; a, b\right)\right) \\
& +H\left(t^{r^{*}}\left(a^{\prime}\right), t^{s^{*}}\left(b^{\prime}\right)\right) .
\end{aligned}
$$

This minimization problem is solved sequentially for each node $\left[t^{r}(a), t^{s}(b)\right]$, starting from $\left[t^{r}(1), t^{s}(1)\right]$, and with $H\left(t^{r}(1), t^{s}(1)\right)=0$, and increasing $a$ and $b$ until the node $\left[t^{r}\left(N_{r}\right), t^{s}\left(N_{s}\right)\right]$ is reached. Tracing the path created by the set of pairs $\left\{\left[t^{*^{*}}(1), t^{s^{*}}(1)\right], \ldots,\left[t^{r^{*}}\left(N_{r}\right), t^{s^{*}}\left(N_{s}\right)\right]\right\}$ provides a discrete version of the optimal continuous $\gamma$. It is important to highlight that the dynamic programming algorithm provides the exact optimal warping function for each node, i.e. the warping function found can never be a local optimum of the objective function, and, therefore, the validity of $d_{w}$ and $d_{a}$ will not be compromised.

In this work, we used the standard dynamic programming algorithm [26] to solve for an optimal $\gamma$ in eq. (3). It should be noted that for any fixed partition of $\boldsymbol{t}^{r}$, this algorithm provides the exact optimal $\gamma$ that is restricted to the graph on this partition. Recently, an optimization method of performing optimization methods on Riemannian manifolds was developed [42], with one method being the Broyden-FletcherGoldfarb-Shanno (BFGS) algorithm. The BFGS algorithm is a faster alternative to dynamic programming. Additionally, an optimization approach using a Dirichlet prior on the warping functions, and a Markov chain Monte Carlo algorithm has been recently developed [43].

\section{APPENDIX B \\ MEAN WARPED T-WAVE}

From a set of $I$ T-waves, $\left\{\boldsymbol{f}_{1}^{s}\left(\boldsymbol{t}_{1}^{s}\right), \boldsymbol{f}_{2}^{s}\left(\boldsymbol{t}_{2}^{s}\right), \ldots, \boldsymbol{f}_{I}^{s}\left(\boldsymbol{t}_{I}^{s}\right)\right\}$ with temporal and amplitude variability it is possible to calculate a mean warped T-wave that is an optimal representative average both in temporal and amplitude domains. Consequently, we will iteratively search for the optimal mean warped Twave in the SRSF domain, $\overline{\boldsymbol{q}}^{k}\left(\boldsymbol{t}^{r}\right)$, where $k$ is the iteration, that minimizes the average of the difference between $\overline{\boldsymbol{q}}^{k}\left(\boldsymbol{t}^{r}\right)$ and each of the SRSF transformations of the set of Twaves, $\left\{\boldsymbol{q}_{f_{1}^{s}}\left(\boldsymbol{t}_{1}^{s}\right), \boldsymbol{q}_{f_{2}^{s}}\left(\boldsymbol{t}_{2}^{s}\right), \ldots, \boldsymbol{q}_{f_{I}^{s}}\left(\boldsymbol{t}_{I}^{s}\right)\right\}$. Therefore, we initialize $\overline{\boldsymbol{q}}^{k=1}\left(\boldsymbol{t}^{r}\right)$ as the average of $\left\{\boldsymbol{q}_{f_{1}^{s}}\left(\boldsymbol{t}_{1}^{s}\right), \boldsymbol{q}_{f_{2}^{s}}\left(\boldsymbol{t}_{2}^{s}\right), \ldots, \boldsymbol{q}_{f_{I}^{s}}\left(\boldsymbol{t}_{I}^{s}\right)\right\}$ and, then, for iteration $k$, we look for the optimal set of $\left\{\boldsymbol{\gamma}_{1}^{*^{k}}\left(\boldsymbol{t}^{r}\right), \boldsymbol{\gamma}_{2}^{*^{k}}\left(\boldsymbol{t}^{r}\right), \ldots, \boldsymbol{\gamma}_{I}^{*^{k}}\left(\boldsymbol{t}^{r}\right)\right\}$ that minimizes:

$$
\begin{gathered}
\boldsymbol{\gamma}_{i}^{*^{k}}\left(\boldsymbol{t}^{r}\right)=\underset{\gamma_{i}\left(\boldsymbol{t}^{r}\right)}{\arg \min }\left(\left\|\overline{\boldsymbol{q}}^{k}\left(\boldsymbol{t}^{r}\right)-\boldsymbol{q}_{f_{i}^{s}\left(\boldsymbol{\gamma}_{i}\right)}\left(\boldsymbol{t}^{r}\right)\right\|\right), \\
i=1, \ldots, I .
\end{gathered}
$$

The updated SRSF transformation of the mean warped Twave can be calculated as:

$$
\overline{\boldsymbol{q}}^{k+1}\left(\boldsymbol{t}^{r}\right)=\frac{1}{I} \sum_{i=1}^{I} \boldsymbol{q}_{\left[f_{i}^{s} \circ \gamma_{i}^{* k}\right]}\left(\boldsymbol{t}^{r}\right)
$$

We repeated eq. (23) and (24) until the difference between the energies of $\boldsymbol{q}^{k+1}\left(\boldsymbol{t}^{r}\right)$ and $\boldsymbol{q}^{k}\left(\boldsymbol{t}^{r}\right)$ was lower than $0.1 \%$. 
Then, the final mean warped T-wave can be obtained as $[23,25]:$

$$
\bar{f}\left(t^{r}(n)\right)=\frac{1}{I} \sum_{i=1}^{I} f_{i}^{s}\left(t_{i}^{s}(1)\right)+\sum_{l=1}^{n} \bar{q}^{k}\left(t^{r}(l)\right)\left|\bar{q}^{k}\left(t^{r}(l)\right)\right|
$$

When the morphology of the set of T-waves is very homogeneous, the morphology of the mean warped T-wave will be very similar and representative of the set. However, if the morphologies in the set are heterogeneous, like when a large portion of T-waves are biphasic or S-shaped while another portion is monophasic, the mean warped T-wave morphology will resemble the dominant T-wave shape, in case there exists one, or the arithmetic T-wave mean (after warping), if the different populations have the same weight. Therefore, it would be advisable to include a pre-processing step to remove any undesired T-wave morphology prior the computation of the mean warped T-wave.

\section{APPENDIX C ECG-SIM}

The transmembrane potentials, $\boldsymbol{v}(t)=\left[v_{1}(t), \ldots, v_{M}(t)\right]$, of $M=257$ sources (nodes), each one located at a given point on the epi- and endocardium, were generated for each node $m$ by using the analytical expression proposed in [44], and already used for similar purposes in [45, 46]:

$$
\begin{aligned}
& v_{m}(t)=a_{m} \cdot \mathfrak{d}_{m}(t) \cdot \mathfrak{r}_{m}(t)+v_{0}, \quad m=1 . . M, \\
& \mathfrak{d}_{m}(t)=\frac{1}{1+e^{-\alpha_{m}\left(t-\delta_{m}\right)}} . \\
& \mathfrak{r}_{m}(t)=\left(1-\frac{1}{1+e^{-\beta_{m}\left(t-\rho_{m}\right)}}\right) \cdot\left(1-\frac{1}{1+e^{-\mu_{m}\left(t-\rho_{m}\right)}}\right) .
\end{aligned}
$$

In these expressions, $\delta_{m}$ and $\rho_{m}$ are the depolarization and repolarization times, and $\alpha_{m}, \beta_{m}$ and $\mu_{m}$ describe the upslope during repolarization, and the leading and trailing downslope during repolarization, respectively, at node $m . a_{m}$ is the amplitude of the transmembrane potential and $v_{0}$ is the resting potential. The values of the parameters were obtained by fitting $v_{m}(t)$ with the transmembrane potential of each node provided by ECGSIM [47] for a normal male.

A $L$-lead ECG beat, with $L=8$, was generated by using the model proposed and validated in [33]. This model is derived from the equivalent surface source model [48], assuming that the sources are of the double layer type. The ECG potential (one for each of the $L$ leads considered) on the body surface can be expressed as:

$$
\phi(t)=\mathbf{A} \boldsymbol{v}(t),
$$

where matrix $\mathbf{A}$ has size $(8 \times M)$ and reflects the complexity of the torso (geometry and conductivity). Each row of $\mathbf{A}$ expresses the linear weighting of all action potentials, $\boldsymbol{v}(t)$, for each standard lead [33].

In [33], the author pointed out that it was possible to link the shape of the T-wave in each lead to the transmembrane potential using equation (27). In fact, making the further approximation that the only difference across different $v_{m}(t)$ functions is the repolarization time $\rho_{m}$, i.e., $v_{m}(t)=$ $v\left(t-\rho_{m}\right)$, then the repolarization time of each node may be expressed as $\rho_{m}=\bar{\rho}+\Delta \rho_{m}$, where $\bar{\rho}=\sum_{m=1}^{M} \rho_{m} / M$ is the average repolarization time, and $\Delta \rho_{m} \ll \bar{\rho}$ is the deviation (difference) of repolarization times at cardiac site $m$, with standard deviation $\sigma$, from the mean repolarization time, with $\sum_{m=1}^{M} \Delta \rho_{m}=0$.

\section{ACKNOWLEDGMENT}

The authors would like to acknowledge the contribution of Dr. R. Sassi for providing the data used at the electrophysiological simulation.

\section{REFERENCES}

[1] R. M. John et al., "Ventricular arrhythmias and sudden cardiac death," Lancet, vol. 380, no. 9852, pp. 1520-1529, 2012.

[2] M. N. Niemeijer et al., "Short-term QT variability markers for the prediction of ventricular arrhythmias and sudden cardiac death: a systematic review," Heart, vol. 100, no. 23, pp. 1831-1836, 2014.

[3] M. Baumert et al., "QT interval variability in body surface ECG: measurement, physiological basis, and clinical value: position statement and consensus guidance endorsed by the European Heart Rhythm Association jointly with the ESC Working Group on Cardiac Cellular Electrophysiology," Europace, euv405, 2016.

[4] W. L. Atiga et al., "Beat-to-beat repolarization lability identifies patients at risk for sudden cardiac death," J. Cardiovasc. Electrophysiol., vol. 9, no. 9, pp. 899-908, 1998.

[5] T. Klingenheben et al., "Predictive value of T-wave alternans for arrhythmic events in patients with congestive heart failure," Lancet, vol. 356, no. 9230, pp. 651-652, 2000.

[6] M. P. Nash et al., "Whole heart action potential duration restitution properties in cardiac patients: a combined clinical and modelling study," Exp. Physiol., vol. 91, no. 2, pp. 339-354, 2006.

[7] R. Coronel et al., "Dispersion of repolarization and arrhythmogenesis," Heart Rhythm, vol. 6, no. 4, pp. 537543, 2009.

[8] M. J. Burgess, "Relation of ventricular repolarization to electrocardiographic T wave-form and arrhythmia vulnerability," Am. J. Physiol., vol. 236, no. 3, pp. H391-H402, 1979.

[9] W. Shimizu and C. Antzelevitch, "Cellular basis for long QT, transmural dispersion of repolarization, and torsade de pointes in the long QT syndrome," J. Electrocardiol., vol. 32, Suppl., pp. 177-184, 1999.

[10] K. Gima and Y. Rudy, "Ionic current basis of electrocardiographic waveforms: a model study," Circ. Res., vol. 90, no. 8, pp. 889-896, 2002.

[11] E. Pueyo et al., "Cardiac repolarization analysis using the surface electrocardiogram,” Philos. Trans. A. Math. Phys. Eng. Sci., vol. 367, no. 1887, pp. 213-233, 2009.

[12] P. Laguna et al., "Techniques for ventricular repolarization instability assessment for the ECG," Proceedings of the IEEE, vol. 104, no. 2, pp. 392-415, 2016.

[13] A. J. Moss, "Measurement of the QT interval and the risk associated with QTc interval prolongation: A review," Am. J. Cardiol., vol. 72, no. 6, pp. B23-B25, 1993.

[14] M. S. Fuller et al., "Estimates of repolarization dispersion from electrocardiographic measurements," Circulation, vol. 102, no. 6, pp. 685-691, 2000.

[15] W. Zareba et al., "Automatic detection of spatial and dynamic heterogeneity of repolarization," J. Electrocardiol., vol. 27, Suppl., pp. 66-72, 1994.

[16] A. Mincholé et al., "Quantification of restitution dispersion from the dynamic changes of the T-wave peak to end, measured at the surface ECG," IEEE Trans. Biomed. Eng., vol. 58, no. 5, pp. 1172-1182, May. 2011 
[17] S. M. Narayan, "T-wave alternans and the susceptibility to ventricular arrhythmias," J. Am. Coll. Cardiol., vol. 47, no. 2, pp. 269-281, 2006.

[18] P. Brugada and J. Brugada, "Right bundle branch block, persistent ST segment elevation and sudden cardiac death: A distinct clinical and electrocardiographic syndrome: a multicenter report," J. Am. Coll. Cardiol., vol. 20, no. 6, pp. 1391-1396, 1992.

[19] B. Acar et al., "Spatial, temporal and wavefront direction characteristics of 12-lead T-wave morphology," Med. Biol. Eng. Comput., vol. 37, no. 5, pp. 574-584, 1999.

[20] D. Cuesta-Frau et al., "Enhanced modified moving average analysis of T-wave alternans using a curve matching method: a simulation study," Med. Biol. Eng. Comput., vol. 47, no. 3, pp. 323-331, 2009.

[21] M. Kotas, "Projective filtering of time warped ECG beats," Comput. Biol. Med., vol. 38, no. 1, pp.127-137, 2008.

[22] M. Schmidt et al., "Two-dimensional warping for onedimensional signals: conceptual framework and application to ECG processing," IEEE Trans Signal Process, vol. 62, no. 21, pp. 5577-5588, Sep. 2014

[23] A. Srivastava et al., "Registration of Functional Data Using Fisher-Rao metric," arXiv preprint, arXiv:1103.3817, 2011.

[24] J. O. Ramsay and X. Li, "Curve registration," J. R. Stat. Soc. Series B Stat. Methodol., vol. 60, no. 2, pp. 351-363, 1998.

[25] J. D. Tucker et al., "Generative models for functional data using phase and amplitude separation," Comput. Stat. Data Anal., vol. 61, pp. 50-66, 2013.

[26] D. P. Bertsekas, "Dynamic programming and optimal control," Belmont, MA: Athena Scientific, vol. 1, no. 2, 1995.

[27] A. Srivastava et al., "Shape analysis of elastic curves in euclidean spaces," IEEE Trans. Pattern Anal. Mach. Intell., vol. 33, no. 7, pp. 1415-1428, Oct. 2010.

[28] P. W. Holland and R. E. Welsch, "Robust regression using iteratively reweighted least-squares," Commun. Stat. Theory Methods, vol. 6, no. 9, pp. 813-827, 1977.

[29] P. Laguna et al., "Automatic detection of wave boundaries in multilead ECG signals: Validation with the CSE database," Comput. Biomed. Res., vol. 27, no. 1, pp. 45-60, 1994.

[30] F. Castells et al., "Principal component analysis in ECG signal processing," EURASIP Journal on Applied Signal Processing, vol. 2007, no. 1, pp. 98-98, 2007.

[31] J. P. Martínez et al., "A wavelet-based ECG delineator: evaluation on standard databases," IEEE Trans. Biomed. Eng., vol. 51, no. 4, pp. 570-581, Apr. 2004.

[32] Task force of the European Society of Cardiology and the North American Society of Pacing and Electrophysiology, "Heart rate variability standards of measurement, physiological interpretation, and clinical use," Eur. Heart. J., vol. 17, pp. 354-381, 1996.

[33] A. van Oosterom, "Genesis of the $\mathrm{T}$ wave as based on an equivalent surface source model," J. Electrocardiol., vol. 34, Suppl, pp. 217-227, 2001.

[34] E. Gil et al., "Photoplethysmography pulse rate variability as a surrogate measurement of heart rate variability during non-stationary conditions," Physiol. Meas., vol. 31, no. 9, pp. 1271-1290, 2010.

[35] D. C. Howell, "Median absolute deviation," Wiley StatsRef: Stat Ref Online, 2005.

[36] J. Ramírez et al., "Automatic SVM classification of sudden cardiac death and pump failure death from autonomic and repolarization ECG markers," J. Electrocardiol., vol. 48, no. 4, pp. 551-557, 2015.

[37] R. D. Berger, "Methodology for automated QT variability measurement," U.S. Patent 08/340, 861 Oct 1st 1996.

[38] G. Piccirillo et al., "QT variability strongly predicts sudden cardiac death in asymptomatic subjects with mild or moderate left ventricular systolic dysfunction: A prospective study," Eur. Heart J., vol. 28, no. 11, pp. 1344-1350, 2007.
[39] R. D. Berger et al., "Beat-to-beat QT interval variability: Novel evidence for repolarization lability in ischemic and nonischemic dilated cardiomyopathy," Circulation, vol. 96, no. 5, pp. 1557-1565, 1997.

[40] H. J. L. M. Vullings et al., "ECG segmentation using time-warping," International Symposium on Intelligent Data Analysis, Eds. Berlin, Germany: Springer, 2006: 275-285.

[41] A. Zifan et al., "Automated segmentation of ECG signals using piecewise derivative dynamic time warping," International Journal of Biological and Life Sciences, vol. 1, pp. 181-185, 2007.

[42] W. Huang et al., "A Broyden Class of Quasi-Newton Methods for Riemannian Optimization," SIAM J. Optimiz., vol. 25, no. 3, pp. 1660-1685, 2015.

[43] W. Cheng et al., "Bayesian Registration of Functions and Curves," Bayesian Anal., vol. 11, no. 2, pp. 447-475, 2016.

[44] P. van Dam et al., "Non-invasive imaging of cardiac activation and recovery," Ann. Biomed. Eng., vol. 37, no. 9, pp. 1739-1756, 2009.

[45] R. Sassi and L. T. Mainardi, "An estimate of the dispersion of repolarization times based on a biophysical model of the ECG," IEEE Trans. Biomed. Eng., vol. 58, no. 12, pp. 3396-3405, Dec. 2011.

[46] M. Orini et al., "Detection of transient, regional cardiac repolarization alternans by time-frequency analysis of synthetic electrograms," Conf. Proc. IEEE Eng. Med. Biol. Soc., pp. 3773-3776, 2013.

[47] A. van Oosterom and T. F. Oosterndorp, "ECGSIM: an interactive tool for studying the genesis of qrst waveforms," Heart, vol. 90, no. 2, pp.165-168, 2004.

[48] D. B. Geselowitz, "Description of cardiac sources in anisotropic cardiac muscle. Application of bidomain model," $J$. Electrocardiol., vol. 25, Suppl., pp. 65-67, 1992. 